Article

\title{
An Integrated Procedure for Ex-Ante Evaluations of Refurbishment Costs in Healthcare Facilities
}

\author{
Raul Berto *(D), Carlo Antonio Stival $\mathbb{D}$ and Paolo Rosato $\mathbb{D}$ \\ Department of Engineering and Architecture, University of Trieste, 34127 Trieste, Italy; cstival@units.it (C.A.S.); \\ paolo.rosato@dia.units.it (P.R.) \\ * Correspondence: rberto@units.it
}

Received: 27 July 2020; Accepted: 3 September 2020; Published: 9 September 2020

\begin{abstract}
This paper focuses on the valuation of refurbishment costs for healthcare facilities. The determination of the more reliable approach for experimental verification is a research topic of great interest, especially because previous literature on the matter is limited. This study examines ex-ante cost valuations in the refurbishment of healthcare buildings while using similarity to estimate the costs that are based on the amount of already accomplished renovations. The methodology involved a desk analysis deter-mining the technical valuation of intervention needs, and similarity coefficient applications providing a refurbishment cost valuation. The application was conducted in the Friuli-Venezia Giulia Region in Italy, where hospitals show structural, layout, and plants deficits with respect to current regulations, and a technical deepening to identify critical issues is required to prepare a multi-year intervention plan. The case study results showed that this procedure requires little initial information to run analyses and its application can support investment budget planning purposes.
\end{abstract}

Keywords: building refurbishment; ex-ante cost valuation; similarity degrees; healthcare facilities

\section{Introduction}

Construction cost ex-ante evaluations is a topic of great practical interest for both the private and public sectors, as a cost estimate is the starting point for any convenience evaluation. For private operators, the construction cost is essential for estimating the expected profit, while, for public operators, it is essential for efficient public financial resource allocations. Normally, a building's construction cost can be estimated through (1) an analytical-reconstructive procedure, which, starting from the detailed building project, identifies the necessary elementary work entities and calculates the total construction cost, or (2) a synthetic-comparative process that identifies the construction market's costs and compares the chosen building with similar buildings to estimate the costs. Although the first procedure has the advantage of considering all of the building's peculiarities, it requires a detailed project and does not consider the discounts usually offered by construction companies. This is aimed at determining the order of magnitude of the monetary amount of the construction cost of the workings [1]. The second procedure begins with a brief description of the needed work and, based on the actual incurred costs for similar buildings, embeds the most probable rebates and contingencies. It is usually used to determine the order of magnitude of the budget [1]. In other words, the analytical procedure does not take into account the uncertainties of establishing contracts and completing the building, while the synthetic procedure is based on a less precise building description. As such, determining which approach is more reliable for experimental verification is a research topic of great interest, especially because previous literature on the matter is limited. The synthetic-comparative approach can produce an ex-ante valuation of the construction cost based on this description, as the executive design is not yet available. Moreover, only this approach can be used when the cost estimate aims to 
support the planning of a multi-annual investment budget. There are several studies that provide parametric costs that can be useful for synthetic estimates of retrofitting intervention. Among these, we mention those proposed by Di Ludovico et al. [2], which focus on reconstructions following seismic events, and Dell'Anna et al. [3], which focused on the energy requalification of residential buildings. The synthetic cost estimation procedures are also used in the evaluation of the benefits that derive from seismic retrofit and integrated seismic and energy interventions $[4,5]$.

The appraisal discipline has developed various approaches in the last century to effectively compare the value of goods with similar, but not identical, characteristics. In appraisal theory, Jev-ons's law of indifference can produce the most probable market or production value estimation: 'when a commodity is perfectly uniform or homogeneous in quality, any portion may be indifferently used in place of an equal portion: hence, in the same market, and at the same moment, all portions must be exchanged at the same ratio' [6]. In other words, they have the same value. The law of indifference is very intuitive and easily applicable when the commodity market is competitive, as the subject characteristics are then identical to those of the goods with known price. However, when the goods are not homogeneous, the application becomes more problematic. In these cases, it is necessary to adopt one or more comparison criteria capable of incorporating the differences between the chosen good and similar ones for which the cost is known.

The methodology has been developed referring the valuation of intervention costs for healthcare facilities in the Friuli-Venezia Giulia region, in the north-east of Italy. Many of these hospitals show structural, layout, and plants deficits with respect to current regulations. This methodology attempts to resolve this relevant problem. Moreover, the Regional Administration has begun a technical deepening on the existing facilities in order to identify the critical issues and prepare a multi-year intervention plan.

Although the concept of similarity is pervasive in appraisal, there are few published contributions that propose similarity measures as a comparison method for assessing an evaluated object and similar objects of known value. Further, to our knowledge, similarity measures have never been used for the appraisal of building construction and refurbishment costs. Thus, in this study, we explore the possibility of applying the law of indifference for a cost ex-ante valuation of building refurbishment interventions. In other words, as we know the renovation costs for the existing buildings (comparable elements), we attempt to estimate the cost of refurbishing a healthcare building based on its similarities with the comparable elements, while using an evaluation model that adopts qualitative and quantitative similarity coefficients. We hypothesize that we can estimate the cost from similar intervention costs, and that the degree of similarity between the intervention itself and similar ones represents the weights that the latter assumes in the assessment. Using the same parameters as the similarity inquiry, we will also provide a quick method for the technical assessment of refurbishment needs through a desk analysis determining the building units and technical systems that are in need of refurbishment.

\section{An Overview of Similarity Coefficients}

Similarity coefficients are normally used to solve assignment, classification, and/or clustering problems in various fields [7,8], such as biology [9,10], ethnology [11], taxonomy [12], image retrieval [13], geology [14], chemistry [15], and ecology [16-18]. Similarity and its inverse distance measures can be classified in various ways according to the data used in qualitative, semi-quantitative, and quantitative studies. Similarity coefficients, which are usually devoted to qualitative data, provide a measure of analogy degrees between 0 and 1. Qualitative similarity measures are based on the qualities present in the considered objects. Obviously, the greater the number of similar qualities, the greater the degree of similarity.

Another important element in the classification of similarity measures concerns the used information's degree of completeness in the measure calculations. In fact, the absence of a certain quality can signify certainly absent or a lack of information on that specific characteristic. In this regard, it is useful to distinguish between the symmetrical and asymmetrical similarity measures. Thus, a distinction must be made between sure and missing data when the information is incomplete. 
Symmetric measures consider absence as certain data, while asymmetric ones consider it as lack of information. In these different cases, one would choose symmetric similarity coefficients and asymmetric similarity coefficients, respectively.

\subsection{Qualitative Similarity Coefficients}

Qualitative similarity coefficients normally adopt a binary coding of the information that is used to calculate the similarity degree. For example, if we consider two objects, $j$ and $k$, and assume $x_{i}$ characteristics, with $i=n$ and where $x_{i}$ is 1 , the object has the $i$-th characteristic; otherwise, it is 0 . In symmetric coefficients, the value 0 represents the sure absence of the feature. Table 1 summarizes the two objects comparison with respect to the $x_{i}$ characteristics:

a: number of qualities present in both objects

$\mathrm{d}$ : number of qualities absent in both objects

b: number of qualities present in object $k$ and absent in object $j$

c: number of qualities present in object $j$ and absent in object $k$.

Table 1. Scheme for symmetric and asymmetric similarity coefficients.

\begin{tabular}{ccc}
\hline \multirow{2}{*}{ Object $k$} & \multicolumn{2}{c}{ Object $j$} \\
\cline { 2 - 3 } & $\mathbf{1}$ & $\mathbf{0}$ \\
\hline $\mathbf{1}$ & $\mathrm{a}$ & $\mathrm{b}$ \\
$\mathbf{0}$ & $\mathrm{c}$ & $\mathrm{d}$ \\
\hline
\end{tabular}

In the symmetric coefficients, if we assume that the $x_{i}$ characteristics completely describe the objects, then the present (1/1: a) and absent (0/0: d) qualities in both objects express their degree of similarity. The most common symmetric coefficients are calculated from the ratio between the number of coincident descriptors $(\mathrm{a}+\mathrm{d})$ and the total number of descriptors $n$. However, they are distinguished by the different weight attributed to the concordant and discordant descriptors.

The simple matching coefficient (1) does not discern between 0 and 1 and it is given by the simple ratio between the number of descriptors with the same value $(a, d)$ and the total number of descriptors (n) [12]. The Rogers \& Tanimoto coefficient (2), a variant of the previous one that doubles the weight of discordances $(b, c)$, considers more similar observations with fewer discordances [19]. Finally, the Sneath \& Sokal coefficient (3) is a further variant of the previous coefficients, as it is conceptually opposite, because it gives a double weight to the concordances $(\mathrm{a}, \mathrm{d})$ and considers more similar observations with the largest number of concordances [20].

$$
\begin{gathered}
\text { Simple Marching } S_{j k}=\frac{a+d}{a+b+c+d} \\
\text { Rogers \& Tanimoto } S_{j k}=\frac{a+d}{a+2 b+2 c+d} \\
\text { Sneath \& Sokal } S_{j k}=\frac{2 a+2 d}{2 a+b+c+2 d}
\end{gathered}
$$

The asymmetric similarity coefficients, as mentioned above, consider the absence of a characteristic as a lack of information. In other words, this method does not exclude the possibility that the characteristic is present. In this case, the various coefficients are calculated from the ratio between the number of features that are present in both objects (a) and the number of characteristics certainly in agreement (a) added to the discordant ones (b, c). Among the asymmetric coefficients, the Jaccard coefficient (S4) is strikingly similar to the simple matching coefficient, but it does not consider the discordances $(\mathrm{d})$ and it represents the ratio between the concordances and the number of non-zero 
values [21]. The Sørensen coefficient (5) gives double weight to the concordances, emphasizing the asymmetric criterion [22]. The last two coefficients are used in data samples with uncertain representativeness due to a lack of information.

$$
\begin{aligned}
\text { Jaccard } S_{j k} & =\frac{a}{a+b+c} \\
\text { Sørensen } S_{j k} & =\frac{2 a}{2 a+b+c}
\end{aligned}
$$

\subsection{Semi-Quantitative and Quantitative Similarity Coefficients}

Semi-quantitative and quantitative similarity coefficients are used when the ordinal or cardinal variables describe the compared objects. The quantitative coefficients are distinguished from the qualitative ones to consider not only the presence or absence of a given characteristic, but also its size. For this reason, they are an interesting method to evaluate similarity with respect to the characteristics that influence the market value or production costs.

The Steinhaus coefficient [23], Rudjichka coefficient [24], and Kulczynski coefficient [25] propose similarity measures, starting from a summation of the minimum values found in the compared objects, but they adopt different normalization procedures.

The Steinhaus coefficient (6) normalizes for the summation of the descriptor values, while the Rudjichka coefficient (7) normalizes for the maximum value of each descriptor. Finally, the Kulczynski coefficient (8) represents the ratio between the summation of the minimum values recorded in the two objects' descriptors and their respective summations.

The quantitative Jaccard coefficient [21], also known as the Tanimoto coefficient [26], the Dice coefficient [27], and the Cosine coefficient, instead, propose similarity coefficients, starting from the summations of the products between each descriptor. Jaccard (9) normalizes the product by summing the squares of the product's diminished descriptors. Dice (10) normalizes the product's double among the descriptors with the sum of the descriptors' squares. Finally, the Cosine measure (11) normalizes the product between the descriptors with the summation's square roots of each descriptor's squares.

$$
\begin{gathered}
\text { Steinhaus } S_{j k}=\frac{2 \sum_{i=1}^{n} \min \left(x_{i j}, x_{i k}\right)}{\sum_{i=1}^{n} x_{i j}+x_{i k}} \\
\text { Rudjichka } S_{j k}=\frac{\sum_{i=1}^{n} \min \left(x_{i j}, x_{i k}\right)}{\sum_{i=1}^{n} x_{i j}+x_{i k}} \\
\text { Kulczynski } S_{j k}=\frac{1}{2}\left[\frac{\sum_{i=1}^{n} \min \left(x_{i j}, x_{i k}\right)}{\sum_{i=1}^{n} x_{i j}}+\frac{\sum_{i=1}^{n} \min \left(x_{i j}, x_{i k}\right)}{\sum_{i=1}^{n} x_{i k}}\right] \\
\text { Jaccard } S_{j k}=\frac{\sum_{i=1}^{n} x_{i j} x_{i k}}{\sum_{i=1}^{n} x_{i j}{ }^{2}+\sum_{i=1}^{n} x_{i k}{ }^{2}-\sum_{i=1}^{n} x_{i j} x_{i k}} \\
\text { Dice } S_{j k}=\frac{2 \sum_{i=1}^{n} x_{i j} x_{i k}}{\sum_{i=1}^{n} x_{i j}{ }^{2}+\sum_{i=1}^{n} x_{i k}{ }^{2}} \\
\text { Cosine } S_{j k}=\frac{\sum_{i=1}^{n} x_{i j} x_{i k}}{\sqrt{\sum_{i=1}^{n} x_{i j}{ }^{2}} \sqrt{\sum_{i=1}^{n} x_{i k}^{2}}}
\end{gathered}
$$

\section{Current Applications of Similarity Coefficients}

Most of the existing literature proposes examining similarity for the selection of comparable items or to implement the adjustment grid method. Isakson [28] developed the Nearest Neighbours Appraisal Technique (NNAT) as both an improvement of, and an alternative to, adjustment grid 
methods for house value estimations. Supposing that all of the properties in a particular market are rank-ordered by value, the subject property's value can be expressed, as in Equation (12), where the weights represent the nearness/similarity of the subject to the comparable properties. The weight associated with each comparable item is calculated, starting from the inverse of the Malahanobis distance [29], which allows for the method to consider, in the similarity's estimation, the presence of correlations among the descriptors. In further applications on different properties (dwelling building, industrial properties, office buildings, and retail, etc.), Isakson [30] pitted the NNAT against the traditional real estate appraisal process and the ordinary least square (OLS). The results showed that the NNAT was significantly more accurate than the other two estimates for retail and other miscellaneous properties, but not for other property types.

$$
\hat{P}_{i}=\sum_{j=1}^{k} W_{i j} P_{j} \text { with } w_{i j}=\frac{\left(1 / \mathrm{D}_{i j}^{2}\right)}{\sum_{i=1}^{k}\left(1 / \mathrm{D}_{i j}^{2}\right)}
$$

where

$\hat{P}_{i}$ is the expected price the of house,

$P_{j}$ is actual the price of the house $j$,

$W_{i j}$ is the nearness of house $j$ to house $i$,

$k$ is the number of comparable items,

$D_{i j}$ is the Mahalanobis distance between property $i$ and $j$.

Other studies assessed the comparable sample's similarity and reliability degrees in the market-oriented appraisal method, particularly the Market Comparison Approach (MCA) [31-34]. These studies introduced two different similarity coefficients, a reliability coefficient and two second level indexes, which combine similarity and reliability measures. The similarity indexes are calculated with respect to the differences between the considered objects' descriptors, while the reliability coefficient is calculated, instead, based on the differences in the correct prices. These studies propose the adoption of these indexes in MCA's reconciliation phase if the corrected price diverge. In particular, they suggest weighting the corrected prices with (i) similarity coefficients if the divergence of the corrected price is related to real estate characteristics, (ii) reliability coefficients if the divergence is related to market prices, or (iii) second level indexes if the divergence is related to both real estate characteristics and market prices.

Kulczycki and Ligas [35] presented a procedure for assessing the similarities between properties when they are described by qualitative attributes. They used a computational procedure to transform a nominal attribute into a corresponding set of dummy binary attributes. Subsequently, they applied different similarity coefficients for qualitative data and compared the results by ranking and grouping the sample properties that were derived from different coefficients. Finally, they determined the best similarity coefficients for real estate market application.

Zyga deepened the relationship between estimate validity and comparable item similarities, showing that estimated prices using statistical approaches often 'do not always meet the requirements of statutory definition of market value' [36]. Moreover, the author stressed the need to apply statistical approaches to similarly preselected data sets with the object of the estimate, because 'for the purpose of real estate appraisal itself, the selection of data is more useful than searching for a price model' [37].

This review shows how, for estimative purposes, incorporating similarity in the evaluation process is fundamental for obtaining good estimates, even in the presence of limited market data. In fact, in the presence of limited random data, statistical approaches are not usable, and mathematical attempts often provide results that contrast the market evidence. For similarity analysis, using a comparison criterion or refining an estimate for MCA can be a useful support tool. The above considerations essentially concern evaluations of building market values, but they can easily be extended to examining construction costs, as this also represents a value that is formed in a market. 


\section{Materials and Methods}

Ex-ante evaluation of a healthcare building's refurbishment costs is important for the administration of public finances, as health spending represents $9.8 \%$ of the GDP in European Union countries and $75 \%$ of it is financed by member states [38]. Furthermore, old buildings with significant structural, energy, and plant deficits require urgent and expensive redevelopment [39]. Therefore, the ability to forecast refurbishment expenditures is of great importance to efficiently plan budgetary commitments. This need is even greater in areas at risk of earthquakes, where it is necessary to guarantee the full functionality of health facilities, especially first aid facilities.

The operational phase implies performing maintenance and refurbishments on built heritage sites due to progressive performance lacks. Thus, building asset management becomes increasingly complicated and requires specific knowledge [40]. In healthcare facilities, building conditions are a challenging task involving data acquisition and performance assessments due to their physical systems, special plants, and diverse functional spaces [41].

In the present research, implementing a technical evaluation of the current building status is crucial, as budget estimation in the refurbishment of a healthcare building requires a quick method to assess building conditions based on relevant key performance indicators [42]. This method, applied by a competent assessor, does not replace the analytic-reconstructive procedure that prepares a detailed project. However, it provides asset managers and decision-makers a lean tool for investigating building conditions and activating appropriate interventions based on visual inspections and document availabilities [43].

The proposed methodology investigates a healthcare facility built in a heritage building and considers the criteria that drive refurbishment, adjustment, and modernization policies for large healthcare facilities [44]. The evaluation methodology aims to achieve two targets:

A. identifying the intervention needs of the considered healthcare context (subject) that has deteriorated according to the standards. These interventions can identify the areas in which it will be necessary to operate to refurbish the subject (Technical Assessment); and,

B. estimating the costs of the subject's refurbishments, made possible by the performance data derived from the first phase (Economic Valuation).

Specifically, we performed the valuation of intervention costs for health facilities in the Friuli-Venezia Giulia region, in north-eastern Italy. Many of these hospitals have structural, layout, and plants deficits with respect to current regulations. Thus, the Regional Administration has begun focusing on the existing facilities in order to identify the critical issues and prepare a multi-year intervention plan.

\subsection{Technical Assessment}

To achieve target A and the research purpose, we developed a Quick Requirement Assessment (QRA) method that can be broken down into the following phases:

1. Assessing refurbishment needs.

2. Defining the appropriate Standard Interventions (SI).

3. Identifying intervention needs through the activation of one or more SIs.

The assessment involves both documentation study and non-invasive evaluations, and it contains a Quick Subject Survey (QSS), a Standard Intervention Definition (SID), and a Quick Technical Evaluation (QTE) (Figure 1). The QRA procedure is based on the hypothesis that a hospital, a structure of the highest complexity, requires a very high maintenance level that is constantly performed according to advanced commissioning standards. Consequently, the QRA assumes that the building construction or extraordinary repairs/refurbishments/re-developments of its subsystems have achieved a verified level of regulatory compliance. The complexities and extent of the envisioned verifications for the accreditation of hospitals justify this hypothesis. 


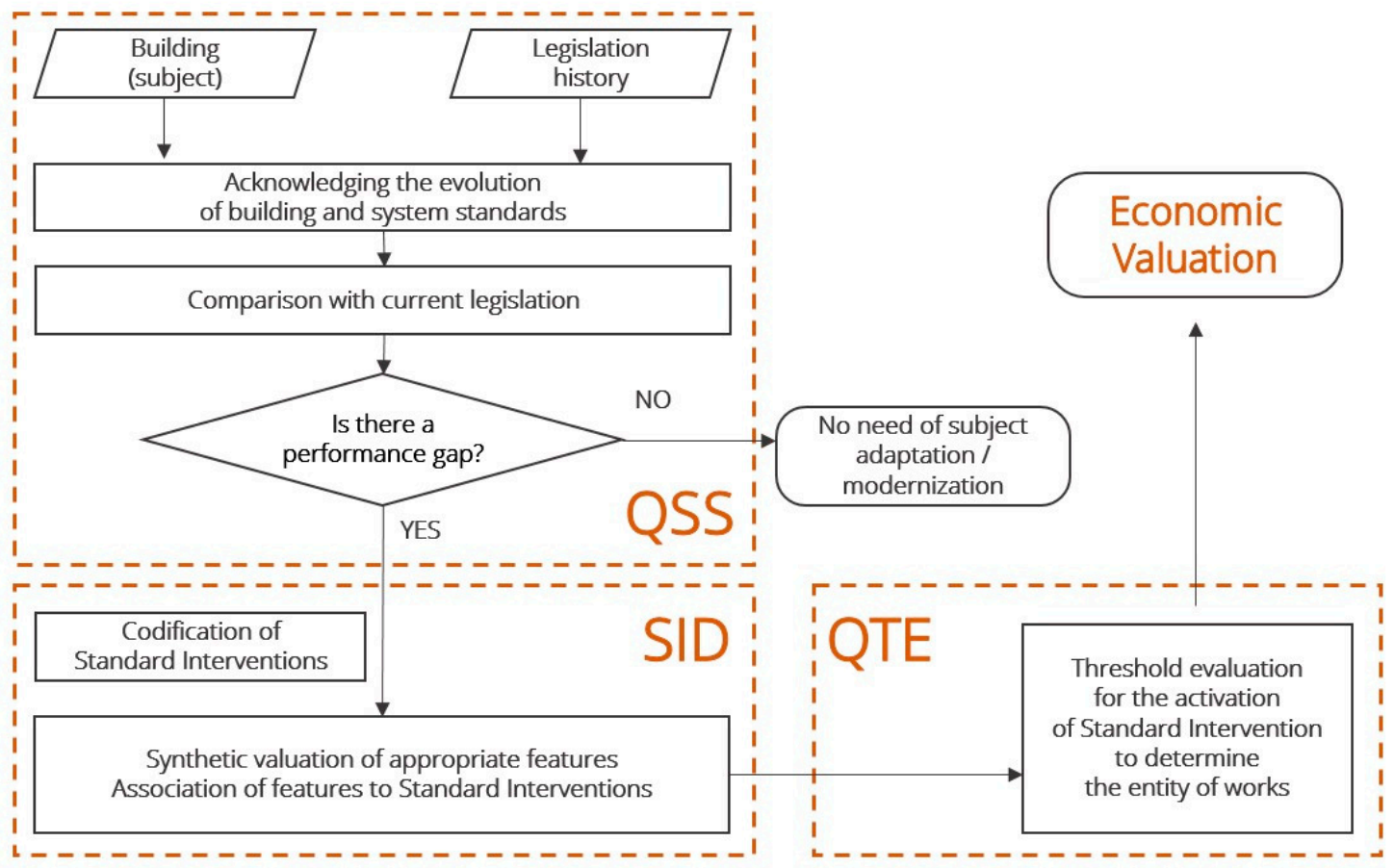

Figure 1. Logical scheme of the Quick Requirement Assessment (QRA) procedure with its subroutines.

\subsubsection{QSS (Subroutine A.1)}

According to the QRA's hypothesis, it is possible to achieve a quick evaluation of the subject's intervention needs. Experienced personnel that have access to the technical documentation behind the subject's construction and total interventions can perform this first subroutine (QSS). The QSS consists of three steps, coded as QSS.1, QSS.2, and QSS.3, and it represents the collection of technical information: subject history reconstruction, site condition evaluations, and integrative technical findings.

The first step, QSS.1, aims to overview the subject's history, highlighting its construction period and all significant interventions concerning its technological systems, given the importance of key hospital equipment [41]. Table 2 lists the significant features that essentially describe the subject through the time periods representing the typological and technological evolutions of hospitals. QSS.1.1 offers the significant dates in which technological developments and regulatory updates had an impact on the hospital's design in the Italian context [45-48].

We determined up to six possible options for each feature QSS.1.2-QSS.1.11, as it is essential to identify the most recent interventions on the subject's spatial and technological systems. Every option refers to the issues of legislative regulations that updated design criteria, and a technological system's implementation and management. It is reasonable to assume that these regulations have driven previous interventions for refurbishment, maintenance, and adaptation. The considered features involve the following:

- $\quad$ structural adjustments and improvements [49-53];

- building envelope refurbishments [54-57];

- HVAC system installations and requalifications [55-58];

- fire-detection system installations and adjustments [59-62];

- electrical and special equipment installations and requalifications [63-65];

- renovation and enhancement of lifting plants and facilities [66,67];

- renovation and enhancement of medical gas plants [68,69];

- indoor layout renovations and adjustments, and architectural barrier removals [70,71]; and,

- sanitary waste treatment and storage area improvements [72-75]. 
Table 2. Section QSS.1: documented history.

\begin{tabular}{|c|c|c|c|c|c|c|}
\hline \multirow{3}{*}{ Features } & \multicolumn{6}{|c|}{ QSS-Section QSS.1 } \\
\hline & \multicolumn{6}{|c|}{ Possible Options } \\
\hline & 1 & 2 & 3 & 4 & 5 & 6 \\
\hline QSS.1.1. Construction period & $<1900$ & 1901-1938 & 1939-1967 & 1968-1996 & 1997-2000 & $>2000$ \\
\hline $\begin{array}{l}\text { QSS.1.2. Latest interventions in structural } \\
\text { adjustments/improvements }\end{array}$ & $<1975$ & 1975-1986 & 1987-1996 & 1997-2003 & 2004-2008 & $>2009$ \\
\hline $\begin{array}{l}\text { QSS.1.3. Latest energy refurbishments of } \\
\text { opaque envelope }\end{array}$ & $<1976$ & 1977-1991 & 1992-2005 & 2006-2015 & $>2015$ & - \\
\hline $\begin{array}{l}\text { QSS.1.4. Latest energy refurbishment of } \\
\text { transparent envelope }\end{array}$ & $<1976$ & 1977-1991 & 1992-2005 & 2006-2015 & $>2015$ & - \\
\hline $\begin{array}{l}\text { QSS.1.5. Installation period or latest } \\
\text { requalification of HVAC systems and plants }\end{array}$ & $<1991$ & 1992-2005 & 2006-2011 & 2012-2015 & $>2015$ & - \\
\hline $\begin{array}{l}\text { QSS.1.6. Installation period or latest } \\
\text { requalification of fire-extinguishing and } \\
\text { fire-detection plants }\end{array}$ & $<1984$ & 1985-2002 & 2003-2012 & 2013-2015 & $>2015$ & - \\
\hline $\begin{array}{l}\text { QSS.1.7. Installation period or latest } \\
\text { requalification of special and electric plants }\end{array}$ & $<1971$ & 1971-1990 & 1991-2008 & $>2008$ & - & - \\
\hline $\begin{array}{l}\text { QSS.1.8. Installation period or latest } \\
\text { requalification of elevators and lift systems }\end{array}$ & $<1997$ & 1997-2005 & 2006-2017 & $>2017$ & - & - \\
\hline $\begin{array}{l}\text { QSS.1.9. Installation period or latest } \\
\text { requalification of medical gas plants }\end{array}$ & $<1997$ & 1997-2010 & 2010-2017 & $>2017$ & - & - \\
\hline $\begin{array}{l}\text { QSS.1.10. Latest intervention for layout } \\
\text { adjustments/architectural barrier removals }\end{array}$ & $<1978$ & 1978-1996 & $>1996$ & - & - & - \\
\hline $\begin{array}{l}\text { QSS.1.11. Latest interventions adjusting the } \\
\text { infrastructures for contaminated waste } \\
\text { disposal and waste storage areas }\end{array}$ & $<1996$ & 1997-2006 & $>2007$ & - & - & - \\
\hline
\end{tabular}

For each feature, we assigned a value between a variable range to each option. The options are valued in accordance with their maximum number (Table 2). A more recent refurbishment/ improvement/adjustment intervention assigns a higher value to the feature and this value increases the more that the subject appears to follow the regulatory requirements for performance levels at the time of the QSS.

Step QSS.2 defines the site conditions of the subject location (seismic risk, climate, rainfall, present use, and emergency risk profiles) and provides functional distribution and structural system categorizations [53,62,76]. Table 3 lists the QSS.2 features and the related options. QSS.2.6, QSS.2.7, and QSS.2.8 are used for comparable intervention selection during the pursuit of target B's economic valuation.

The QSS.3 step allocates additional information to complete the intervention needs framework (Table 4).

Table 3. Section QSS.2: integrative information concerning the status quo.

\begin{tabular}{|c|c|c|c|c|c|c|}
\hline \multirow{2}{*}{ Features } & \multicolumn{3}{|c|}{ QSS-Section QSS.2 } & & & \\
\hline & 1 & 2 & 3 & 4 & 5 & 6 \\
\hline QSS.2.2. Heat degree, days $*\left[{ }^{\circ} \mathrm{C}\right]$ & $<2200^{\circ}$ & $2200-2400$ & $2400-2600$ & $>2600$ & - & - \\
\hline $\begin{array}{l}\text { QSS.2.3. Dry periods, } 5 \text {-mm-cumulative } \\
\text { rainy days per year* }[\mathrm{mm}]\end{array}$ & $<60$ & $60-75$ & $>75$ & - & - & - \\
\hline QSS.2.5. Activity risk profile ** & $\mathrm{A} 2-\mathrm{A} 3$ & B & Ciii3 & D1 & D2 & - \\
\hline QSS.2.6. Intended use of the space/area & Facilities & Visitors/Residence & Ambulatory clinic & Laboratory & Patient & Emergency \\
\hline QSS 2.7. Structural typology & Masonry & Concrete & Steel & Composite & - & - \\
\hline QSS 2.8. Functional typology & Mono-block & Poly-block & Court & Tower & Slab & Backbone \\
\hline
\end{tabular}

Notes: * depends on building location; ${ }^{* *}$ stated with reference to D.M. 3 August 2015. With reference to activity risk profile assessments, the letter within the alphanumeric code identifies occupants' characterizations: (A) occupants who are awake and aware of the activities being held, (B) occupants who are not aware of the activities being held, (C) occupants who may be asleep, and (D) patients. The number identifies the fire growth rate, proportionally. 
Table 4. Section QSS.3: integrative information concerning the status quo.

\begin{tabular}{|c|c|c|c|c|}
\hline \multicolumn{5}{|c|}{ QSS-Section QSS.3 } \\
\hline \multirow{2}{*}{ Features } & \multicolumn{4}{|c|}{ Options } \\
\hline & 1 & 2 & 3 & 4 \\
\hline QSS.3.1. Balanced mechanical ventilation & None & & & Installed \\
\hline $\begin{array}{l}\text { QSS.3.2. Renewable energy systems for } \\
\text { thermal energy production }\end{array}$ & None & & & Installed \\
\hline $\begin{array}{l}\text { QSS.3.3. Renewable energy systems for } \\
\text { electricity production }\end{array}$ & None & & & Installed \\
\hline $\begin{array}{l}\text { QSS.3.4. Efficiency of thermal energy } \\
\text { production system }\end{array}$ & $<0.85$ & $0.85-0.95$ & $>0.95$ & $\begin{array}{l}\text { Derived by RES } \\
\text { for at least } 20 \%\end{array}$ \\
\hline QSS.3.5. Efficiency of DHW production system & $<0.80$ & $0.80-0.90$ & $>0.90$ & $\begin{array}{l}\text { Derived by RES } \\
\text { for at least } 50 \%\end{array}$ \\
\hline $\begin{array}{l}\text { QSS.3.6. Dangerous materials in outdoor } \\
\text { spaces/areas }\end{array}$ & Absent & & & Present \\
\hline $\begin{array}{l}\text { QSS.3.7. Dangerous materials in indoor } \\
\text { spaces/areas }\end{array}$ & Absent & & & Present \\
\hline $\begin{array}{l}\text { QSS.3.8. Availability of relevant parking areas } \\
\text { compared to gross cubic volume }\left[\mathrm{m}^{2} \mathrm{~m}^{-3}\right]\end{array}$ & $\leq 0.8$ & & & $>0.8$ \\
\hline
\end{tabular}

The QSS subroutines use 27 features that were defined by two to six value options. Their valorization offers an indicator that can be used to define the subject's intervention needs.

\subsubsection{SID (Subroutine A.2)}

The QSS subroutine provides data that must be structured in a framework that can perform an intervention's economic valuation. In the SID, we proposed a set of 38 SIs. Each SI is independent, while being applicable to every subject, and it involves a combination of construction works, whose coordination satisfies an intervention need or a criticality of one or more building sub-systems.

Three attribute categories characterize each SI.

1. Scope: the need within the SI to perform its purpose. SIs are grouped into seven scopes.

2. Relevant categories of construction works.

3. Partial allocation coefficients for each category, defined according to an expert-based approach. The coefficients represent a category's technical burden that contributes to performing the SI.

A SI consists of at least one main construction work category and, where applicable, ancillary categories to perform a complete and functional intervention. Table 5 shows the SIs and their related scopes.

Table 5. SI framework defined during the Standard Intervention Definition (SID) subroutine.

\begin{tabular}{cccl}
\hline Scope Codes & Scopes & SI Codes & \multicolumn{1}{c}{ Standard Interventions (SI) } \\
\hline \multirow{4}{*}{ SS } & SS1 & Global seismic reinforcement \\
& SS2 & Seismic adjustment of HVAC systems and plants \\
& & SS3 & Seismic adjustment of sanitary and fire-extinguishing plants \\
& & SS4 & Seismic adjustment of medical gas plants \\
& SS5 & Seismic adjustment of special and electric plants \\
\hline \multirow{3}{*}{ FS } & FS1 & New fire compartmentalization/fire protection works \\
& Fire Safety & FS2 & Installation of fire-extinguishing hydrant plant/network \\
& & FS3 & Installation of fire-extinguishing sprinkler plant/network \\
& FS4 & Sectioning of medical gases network \\
& FS5 & Installation of fire-detection plant \\
& FS6 & Installation of fire escape elevator \\
\hline \multirow{2}{*}{ LS } & FS1 & Improvement of storm water disposal system \\
& \multirow{3}{*}{ Flooding Safety } & LS2 & Flooding protection works \\
& & LS3 & Installation of flooding protection pumps \\
\hline
\end{tabular}


Table 5. Cont.

\begin{tabular}{|c|c|c|c|}
\hline Scope Codes & Scopes & SI Codes & Standard Interventions (SI) \\
\hline \multirow{11}{*}{$\mathrm{RF}$} & \multirow{11}{*}{$\begin{array}{l}\text { Routine } \\
\text { Functionality }\end{array}$} & RF1 & Works for architectural barrier removals \\
\hline & & RF2 & Functional adjustment of indoor and outdoor spaces \\
\hline & & RF3 & Substitution of HVAC systems and plants \\
\hline & & RF4 & Modification of HVAC systems and plants \\
\hline & & RF5 & Substitution of sanitary and fire-extinguishing plants \\
\hline & & RF6 & Modification of thermal sanitary and fire-extinguishing plants \\
\hline & & RF7 & Substitution of medical gas plants \\
\hline & & RF8 & Modification of medical gas plants \\
\hline & & RF9 & Substitution of special and electric plants \\
\hline & & RF10 & Modification of special and electric plants \\
\hline & & RF11 & Lift system adjustment \\
\hline \multirow{5}{*}{$\mathrm{EE}$} & \multirow{5}{*}{$\begin{array}{l}\text { Energy } \\
\text { Efficiency }\end{array}$} & EE1 & Thermal insulation of opaque building envelope \\
\hline & & EE2 & Enhancement of transparent building envelope \\
\hline & & EE3 & New HVAC systems and plants for higher efficiency \\
\hline & & EE4 & New DHW production systems for higher efficiency \\
\hline & & EE5 & New power production systems for higher efficiency \\
\hline \multirow{7}{*}{ ES } & \multirow{7}{*}{$\begin{array}{l}\text { Environmental } \\
\text { Sustainability }\end{array}$} & ES1 & $\begin{array}{l}\text { Improvement of infrastructures for contaminated waste } \\
\text { disposal }\end{array}$ \\
\hline & & ES2 & Improvement of outdoor areas for waste storage \\
\hline & & ES3 & Removal of dangerous materials from outdoor spaces \\
\hline & & ES4 & Removal of dangerous materials from indoor spaces \\
\hline & & ES5 & $\begin{array}{l}\text { Installation of renewable energy system for thermal energy } \\
\text { production }\end{array}$ \\
\hline & & ES6 & $\begin{array}{l}\text { Installation of renewable energy system for electricity } \\
\text { production }\end{array}$ \\
\hline & & ES7 & Installation of a storage and reuse system for rainwater \\
\hline FA & Facilities & FA1 & New parking areas \\
\hline
\end{tabular}

Table 6 explains the construction work categories and their relevant units of measure, while Tables 7-9 report the full set of partial allocation coefficients in all SIs. The sum of the partial allocation coefficients may exceed 1.00 for each SI.

Table 6. Construction work categories considered in the technical assessment and relevant measure units.

\begin{tabular}{|c|c|c|}
\hline Category Codes & Construction Works Categories & Measure Units for each Category \\
\hline A & Excavations & $\mathrm{m}^{2}$-building sediment \\
\hline $\mathrm{B}$ & Foundation structures & $\mathrm{m}^{2}$-building sediment \\
\hline $\mathrm{C}$ & Elevation structures & $\mathrm{m}^{3}$-building gross volume \\
\hline $\mathrm{D}$ & Thermal systems and HVAC plants & $\mathrm{m}^{3}$-building gross volume \\
\hline $\mathrm{E}$ & DHW production, sanitary, active fire protection systems & $\mathrm{m}^{2}$-building gross area \\
\hline $\mathrm{F}$ & Medical gas plants and distribution networks & $\mathrm{m}^{2}$-building gross area \\
\hline G & Power plants, electric systems, and special installations & $\mathrm{m}^{2}$-building gross area \\
\hline $\mathrm{H}$ & Lift systems & $\mathrm{m}^{3}$-building gross volume \\
\hline $\mathrm{I}$ & Floorings and floor roughs & $\mathrm{m}^{2}$-building gross area \\
\hline $\mathrm{J}$ & Indoor wall finishes & $\mathrm{m}^{3}$-building gross volume \\
\hline K & Vertical envelope closures and indoor partitions & $\mathrm{m}^{3}$-building gross volume \\
\hline $\mathrm{L}$ & External frames, fixtures, and glazing & $\mathrm{m}^{2}$-opaque envelope surface \\
\hline M & Envelope thermal insulation & $\mathrm{m}^{2}$-glazing envelope surface \\
\hline $\mathrm{N}$ & External finishes & $\mathrm{m}^{2}$-building envelope surface \\
\hline $\mathrm{O}$ & Ceilings & $\mathrm{m}^{2}$-building gross area \\
\hline $\mathrm{P}$ & Internal frames, fixtures, and glazing & $\mathrm{m}^{3}$-building gross volume \\
\hline Q & Roofing & $\mathrm{m}^{2}$-building sediment \\
\hline $\mathrm{R}$ & Outdoor works & $\mathrm{m}^{3}$-building gross volume \\
\hline S & Dangerous material removals & $\mathrm{m}^{3}$-building gross volume \\
\hline $\mathrm{T}$ & Parking & stall \\
\hline
\end{tabular}


Table 7. Construction work categories considered in the technical assessment and relevant measure units for Seismic Safety, Fire Safety, and Flooding Safety scopes.

\begin{tabular}{|c|c|c|c|c|c|c|c|c|c|c|c|c|c|c|}
\hline \multirow{3}{*}{$\begin{array}{l}\text { Category } \\
\text { Codes }\end{array}$} & \multicolumn{14}{|c|}{ Scopes/SI Codifications (SID) } \\
\hline & \multicolumn{5}{|c|}{ SS } & \multicolumn{6}{|c|}{ FS } & \multicolumn{3}{|c|}{ LS } \\
\hline & 1 & 2 & 3 & 4 & 5 & 1 & 2 & 3 & 4 & 5 & 6 & 1 & 2 & 3 \\
\hline A & 0.15 & - & - & - & - & - & - & - & - & - & - & 0.05 & 0.05 & - \\
\hline B & 0.20 & - & - & - & - & - & - & - & - & - & - & - & - & - \\
\hline C & 0.45 & - & - & - & - & - & - & - & - & - & - & - & - & - \\
\hline $\mathrm{D}$ & - & 0.05 & - & - & - & - & - & - & - & - & - & - & - & - \\
\hline E & - & - & 0.05 & - & - & - & 0.30 & 0.30 & - & 0.30 & - & - & - & 0.05 \\
\hline F & - & - & - & 0.05 & - & - & - & - & 0.10 & - & - & - & - & - \\
\hline G & - & - & - & - & 0.05 & - & - & - & - & - & 0.10 & - & - & - \\
\hline $\mathrm{H}$ & - & - & - & - & - & - & - & - & - & - & 0.30 & - & - & - \\
\hline I & 0.05 & 0.05 & 0.05 & 0.05 & 0.05 & 0.05 & 0.05 & 0.05 & 0.05 & 0.05 & 0.05 & - & 0.25 & - \\
\hline $\mathrm{J}$ & 0.05 & 0.05 & 0.05 & 0.05 & 0.05 & 0.20 & 0.05 & 0.05 & 0.05 & 0.05 & 0.05 & - & 0.15 & - \\
\hline K & - & - & 0.05 & - & - & 0.25 & 0.05 & - & - & 0.05 & 0.15 & - & 0.15 & - \\
\hline L & - & - & - & - & - & - & - & - & - & - & - & - & 0.10 & - \\
\hline $\mathrm{M}$ & 0.10 & - & - & - & - & - & - & - & - & - & - & - & - & - \\
\hline $\mathrm{N}$ & 0.10 & - & - & - & - & - & - & - & - & - & - & - & 0.20 & - \\
\hline $\mathrm{O}$ & 0.10 & 0.05 & 0.05 & 0.05 & 0.05 & 0.10 & 0.05 & 0.05 & 0.05 & 0.05 & 0.05 & - & - & - \\
\hline $\mathrm{P}$ & - & - & & - & - & 0.10 & - & - & - & - & 0.10 & - & - & - \\
\hline $\mathrm{Q}$ & - & - & - & - & - & - & - & - & - & - & - & 0.10 & 0.20 & - \\
\hline$\hat{\mathrm{R}}$ & - & - & - & - & - & - & - & - & - & - & - & 0.15 & - & - \\
\hline S & - & - & - & - & - & - & - & - & - & - & - & - & - & - \\
\hline $\mathrm{T}$ & - & - & - & - & - & - & - & - & - & - & - & - & - & - \\
\hline $\begin{array}{l}\text { SI Overall } \\
\text { Intensity }\end{array}$ & 1.20 & 0.20 & 0.25 & 0.20 & 0.20 & 0.70 & 0.50 & 0.45 & 0.25 & 0.50 & 0.80 & 0.30 & 1.10 & 0.05 \\
\hline
\end{tabular}

Table 8. Construction work categories considered in the technical assessment and relevant measure units for Routine Functionality scope.

\begin{tabular}{|c|c|c|c|c|c|c|c|c|c|c|c|}
\hline \multirow{3}{*}{$\begin{array}{l}\text { Category } \\
\text { Codes }\end{array}$} & \multicolumn{11}{|c|}{ Scopes/SI Codifications (SID) } \\
\hline & \multicolumn{11}{|c|}{ RF } \\
\hline & 1 & 2 & 3 & 4 & 5 & 6 & 7 & 8 & 9 & 10 & 11 \\
\hline A & 0.05 & - & - & - & - & - & - & - & - & - & - \\
\hline $\mathrm{B}$ & 0.05 & - & - & - & - & - & - & - & - & - & - \\
\hline $\mathrm{C}$ & - & - & - & - & - & - & - & - & - & - & - \\
\hline $\mathrm{D}$ & - & 0.10 & 1.00 & 0.50 & - & - & - & - & - & - & - \\
\hline $\mathrm{E}$ & - & 0.10 & - & - & 1.00 & 0.50 & - & - & - & - & - \\
\hline $\mathrm{F}$ & - & 0.10 & - & - & - & - & 1.00 & 0.50 & - & - & - \\
\hline $\mathrm{G}$ & - & 0.10 & - & - & - & - & - & - & 1.00 & 0.50 & - \\
\hline $\mathrm{H}$ & 1.00 & - & - & - & - & - & - & - & - & - & 1.00 \\
\hline I & - & 0.10 & 0.05 & 0.05 & 0.05 & 0.05 & 0.05 & 0.05 & 0.05 & 0.05 & - \\
\hline $\mathrm{J}$ & - & 0.50 & 0.05 & 0.05 & 0.05 & 0.05 & 0.10 & 0.10 & 0.05 & 0.05 & - \\
\hline $\mathrm{K}$ & - & 0.40 & - & - & - & - & - & - & - & - & - \\
\hline $\mathrm{L}$ & - & - & - & - & - & - & - & - & - & - & - \\
\hline M & - & - & - & - & - & - & - & - & - & - & - \\
\hline $\mathrm{N}$ & - & - & - & - & - & - & - & - & - & - & - \\
\hline $\mathrm{O}$ & - & 0.10 & 0.10 & 0.05 & 0.10 & 0.10 & 0.05 & 0.05 & 0.10 & 0.10 & 0.05 \\
\hline $\mathrm{P}$ & - & 0.50 & - & - & - & - & - & - & - & - & - \\
\hline $\mathrm{Q}$ & - & - & - & - & - & - & - & - & - & - & - \\
\hline $\mathrm{R}$ & 0.05 & - & - & - & - & - & - & - & - & - & - \\
\hline$S$ & - & - & - & - & - & - & - & - & - & - & - \\
\hline $\mathrm{T}$ & - & - & - & - & - & - & - & - & - & - & - \\
\hline $\begin{array}{l}\text { SI Overall } \\
\text { Intensity }\end{array}$ & 1.15 & 2.00 & 1.20 & 0.65 & 1.20 & 0.70 & 1.20 & 0.70 & 1.20 & 0.70 & 1.05 \\
\hline
\end{tabular}

As the database is obtained from new construction interventions, existing building refurbishments assume that the technical burdens and costs may be higher than for new buildings. The SI's overall intensity represents the sum of the partial coefficients. 
Table 9. Construction work categories considered in the technical assessment and relevant measure units for Energy Efficiency, Environmental Sustainability, and Facilities scopes.

\begin{tabular}{|c|c|c|c|c|c|c|c|c|c|c|c|c|c|}
\hline \multirow{3}{*}{$\begin{array}{c}\text { Category } \\
\text { Codes }\end{array}$} & \multicolumn{13}{|c|}{ Scopes/SI Codifications (SID) } \\
\hline & \multicolumn{5}{|c|}{ EE } & \multicolumn{7}{|c|}{ ES } & \multirow{2}{*}{$\begin{array}{c}\text { FA } \\
\mathbf{1}\end{array}$} \\
\hline & 1 & 2 & 3 & 4 & 5 & 1 & 2 & 3 & 4 & 5 & 6 & 7 & \\
\hline A & - & - & - & - & - & - & - & - & - & - & - & 0.05 & 0.025 \\
\hline B & - & - & - & - & - & - & - & - & - & - & - & - & - \\
\hline $\mathrm{C}$ & - & - & - & - & - & - & - & - & - & - & - & - & - \\
\hline $\mathrm{D}$ & - & - & 0.30 & - & - & - & - & - & - & 0.15 & - & - & - \\
\hline $\mathrm{E}$ & - & - & - & 0.15 & - & - & - & - & - & - & - & 0.10 & - \\
\hline $\mathrm{F}$ & - & - & - & - & - & - & - & - & - & - & - & - & - \\
\hline G & - & - & - & - & 0.40 & - & - & - & - & - & 0.15 & - & 0.15 \\
\hline $\mathrm{H}$ & - & - & - & - & - & - & - & - & - & - & - & - & - \\
\hline I & - & - & - & - & - & - & - & - & 0.10 & - & - & - & 0.075 \\
\hline $\mathrm{J}$ & - & 0.05 & - & - & - & - & - & - & 0.20 & - & - & - & - \\
\hline $\mathrm{K}$ & - & 0.05 & - & - & - & - & - & - & - & - & - & - & - \\
\hline $\mathrm{L}$ & 0.05 & 1.50 & - & - & - & - & - & - & - & - & - & - & - \\
\hline $\mathrm{M}$ & 1.20 & - & - & - & - & - & - & - & - & - & - & - & - \\
\hline $\mathrm{N}$ & - & 0.05 & - & - & - & - & - & - & - & - & - & - & - \\
\hline $\mathrm{O}$ & - & - & - & - & - & - & - & - & - & - & - & - & - \\
\hline $\mathrm{P}$ & - & - & - & - & - & - & - & - & - & - & - & - & - \\
\hline $\mathrm{Q}$ & 0.25 & - & - & - & - & - & - & - & - & - & - & 0.10 & - \\
\hline $\mathrm{R}$ & - & - & - & - & - & 0.20 & 0.20 & - & - & - & - & 0.05 & 0.05 \\
\hline S & - & - & - & - & - & - & - & 1.00 & 1.00 & - & - & - & - \\
\hline $\mathrm{T}$ & - & - & - & - & - & - & - & - & - & - & - & - & 0.70 \\
\hline $\begin{array}{l}\text { SI Overall } \\
\text { Intensity }\end{array}$ & 1.50 & 1.65 & 0.30 & 0.15 & 0.40 & 0.20 & 0.20 & 1.00 & 1.30 & 0.15 & 0.15 & 0.30 & 1.00 \\
\hline
\end{tabular}

\subsubsection{QTE (Subroutine A.3)}

The goal of the last subroutine is to define ways to activate one or more SIs through intervention need feedback. In the QTE subroutine, we organized the information that was collected in the QSS and associated it to specific SIs. Tables 10-12 report all of the matches among the QSS features and SIs, along with the essential parameters for model activation. In accordance with Tables 2-4, the following information is assigned to each SI:

- A $V_{H}$ score equal to the QSS.1.1 feature score, depending on the subject construction period and varying in range [1-6]. The maximum attributable score is $V_{H, \max }=6$ and the QSS.1.1 feature values are common to all SIs.

- A feature-specific $V_{I}$ score that characterizes a SI (Table 10). Similar to the QSS.1.1 scores, each SI has a maximum value $V_{I, \max }$, depending on the option number declared for the specific QSS feature.

For each SI, we defined an Effective Score $S_{E}$ based on this formula:

$$
S_{E}=0.5 \cdot\left[(1+\lambda) \cdot V_{H}\right] \cdot\left[(1-\lambda) \cdot V_{I}\right] \cdot\left(1-F_{C}\right)
$$

where $\lambda$ is a coefficient that allows us to consider the intervention's flexibility and $F_{C}$ is a correction factor for the site conditions. 
Table 10. Essential information on the Quick Technical Evaluation (QTE) subroutine based on Quick Subject Survey (QSS) results and SID codifications (Seismic Safety, Fire Safety, and Flooding Safety scopes).

\begin{tabular}{|c|c|c|c|c|c|c|c|c|c|c|c|c|c|c|}
\hline \multirow{3}{*}{ Indicators } & \multicolumn{14}{|c|}{ Scopes/SI Codifications } \\
\hline & \multicolumn{5}{|c|}{ SS } & \multicolumn{6}{|c|}{ FS } & \multicolumn{3}{|c|}{ LS } \\
\hline & 1 & 2 & 3 & 4 & 5 & 1 & 2 & 3 & 4 & 5 & 6 & 1 & 2 & 3 \\
\hline $\begin{array}{c}V_{H, \max } \\
V_{H}\end{array}$ & \multicolumn{14}{|c|}{$\begin{array}{c}6 \\
\text { Choices among the options in the OSS.1.1 feature }\end{array}$} \\
\hline $\begin{array}{c}\text { Specific } \\
\text { QSS feature }\end{array}$ & 1.2 & 1.5 & 1.6 & 1.9 & 1.7 & 1.6 & 1.6 & 1.6 & 1.9 & 1.6 & 1.6 & 2.3 & 2.3 & 2.3 \\
\hline$V_{I, \min }$ & 1 & 1 & 1 & 1 & 1 & 1 & 1 & 1 & 1 & 1 & 1 & 1 & 1 & 1 \\
\hline$V_{I, \max }$ & 6 & 5 & 5 & 4 & 4 & 5 & 5 & 5 & 4 & 5 & 5 & 3 & 3 & 3 \\
\hline $\begin{array}{l}\text { Correction } \\
\text { factor on QSS } \\
\text { feature }\end{array}$ & 2.1 & 2.1 & 2.1 & 2.1 & 2.1 & 2.5 & 2.5 & 2.5 & - & 2.5 & 2.5 & 2.4 & 2.4 & 2.4 \\
\hline$F_{C}$ & 0.15 & 0.15 & 0.15 & 0.15 & 0.15 & 0.25 & 0.15 & 0.15 & - & 0.15 & 0.15 & 0.20 & 0.20 & 0.20 \\
\hline
\end{tabular}

Table 11. Essential information on the QTE subroutine based on QSS results and SID codifications (Routine Functionality scope).

\begin{tabular}{|c|c|c|c|c|c|c|c|c|c|c|c|}
\hline \multirow{3}{*}{ Indicators } & \multicolumn{11}{|c|}{ Scopes/SI Codifications } \\
\hline & \multicolumn{11}{|c|}{ RF } \\
\hline & 1 & 2 & 3 & 4 & 5 & 6 & 7 & 8 & 9 & 10 & 11 \\
\hline $\begin{array}{c}V_{H, \max } \\
V_{H}\end{array}$ & \multicolumn{11}{|c|}{$\begin{array}{c}6 \\
\text { Choices among the options in the QSS.1.1 feature }\end{array}$} \\
\hline $\begin{array}{l}\text { Specific } \\
\text { OSS feature }\end{array}$ & 1.10 & 1.10 & 1.5 & 1.5 & 1.6 & 1.6 & 1.9 & 1.9 & 1.7 & 1.7 & 1.8 \\
\hline$V_{I, \min }$ & 1 & 1 & 1 & 1 & 1 & 1 & 1 & 1 & 1 & 1 & 1 \\
\hline$V_{I, \max }$ & 3 & 3 & 5 & 5 & 5 & 5 & 4 & 4 & 4 & 4 & 4 \\
\hline $\begin{array}{l}\text { Correction } \\
\text { factor on QSS } \\
\text { feature }\end{array}$ & - & - & 3.1 & 1.5 & 2.5 & 2.5 & - & - & - & - & - \\
\hline$F_{C}$ & - & - & 0.15 & - & 0.25 & - & - & - & - & - & - \\
\hline
\end{tabular}

Table 12. Essential information on the QTE subroutine based on QSS results and SID codifications (Energy Efficiency, Environmental Sustainability and Facilities scopes).

\begin{tabular}{|c|c|c|c|c|c|c|c|c|c|c|c|c|c|}
\hline \multirow{3}{*}{ Indicators } & \multicolumn{13}{|c|}{ Scopes/SI Codifications } \\
\hline & \multicolumn{5}{|c|}{ EE } & \multicolumn{7}{|c|}{ ES } & \multirow{2}{*}{$\begin{array}{r}\text { FA } \\
1\end{array}$} \\
\hline & 1 & 2 & 3 & 4 & 5 & 1 & 2 & 3 & 4 & 5 & 6 & 7 & \\
\hline$V_{H, \max }$ & \\
\hline$V_{H}$ & \multicolumn{13}{|c|}{ Choices among the options in the QSS.1.1 feature } \\
\hline $\begin{array}{l}\text { Specific } \\
\text { QSS feature }\end{array}$ & 1.3 & 1.4 & 3.4 & 3.5 & 1.7 & 1.11 & 1.11 & 3.6 & 3.7 & 1.5 & 3.3 & 2.3 & 3.8 \\
\hline$V_{I, \min }$ & 1 & 1 & 1 & 1 & 1 & 1 & 1 & 0 & 0 & 1 & 0 & 1 & 0 \\
\hline$V_{I, \max }$ & 5 & 5 & 4 & 4 & 4 & 3 & 3 & 1 & 1 & 5 & 1 & 3 & 1 \\
\hline $\begin{array}{l}\text { Correction } \\
\text { factor on QSS } \\
\text { feature }\end{array}$ & 2.2 & 2.2 & - & - & - & - & - & - & - & 3.2 & - & - & - \\
\hline$F_{C}$ & 0.3 & 0.3 & 0.3 & 0.3 & 0.3 & - & - & - & - & 0.15 & - & - & - \\
\hline
\end{tabular}

Coefficient $\lambda$ varies in range [-0.5-0.5] for each SI and considers the relationship between the QSS.1.1 and the SI specific features. With $\lambda=0$, the subject construction and the last adjustment/renovation intervention periods have the same importance. If $\lambda>0$, the construction typology, functional distribution, and system layout prevail over any further interventions. Thus, a renovation/adjustment intervention can hardly overcome the original subject's peculiarities. This applies to global structural seismic reinforcements (SS1) or architectural barrier removals (RF1). When $\lambda<0$, previous interventions on a specific subsystem complied with the refurbishment needs, 
thereby reducing the probability of a new intervention. For example, we can attribute a $\lambda<0$ to flood protection plant installations or building envelope improvements. In general, $\lambda$ tends to be 0.5 the more recent the construction or the higher the attributed value to the QSS feature, specifically the considered SI. Conversely, $\lambda$ tends to be -0.5 the older the subject and the more dated the relevant historical interventions.

The $F_{C}$ correction factor considers the site conditions (step QSS.2) that may make it more desirable to activate a SI. For example, a higher peak ground acceleration value increases the need for seismic adjustments (SS2-SS5). Further, a higher probability of intense rainfall increases the flooding risk and, consequently, the activation probability of SIs that belong to the LS scope.

For each SI, we defined a Reference Score $S_{R}$ :

$$
S_{R}=0.5 \cdot V_{H, \max } \cdot V_{I, \max }
$$

It shows the conditions of a new building, with regular maintenance, in which the maximum possible value of each feature is reached. An SI's activation occurs if

$$
S_{E}<S_{R} \cdot F_{B}=S_{A}
$$

where $F_{B}<1$ is a benchmark factor and $S_{A}$ is the Standard Activation Score. With $F_{B}$, the expert evaluator can appropriately reduce the $S_{R}$, thereby obtaining an $S_{A}$. Without an evaluator's autonomous decision, the $F_{B}$ can be expressed, as follows:

$$
F_{B}=\left(\frac{1}{2} \cdot \frac{v_{H, \max }-v_{H}}{v_{H, \max }}\right)+\left(\frac{1}{2} \cdot \frac{v_{I, \max }-v_{I}}{v_{I, \max }}\right)
$$

The entire QRA procedure allows for the activation of SIs corresponding to the surveyed intervention needs. Each SI offers specific partial coefficients of allocation for one or more construction work categories that define SI intensity. An expert evaluation of spatial dimensions that characterize refurbishment needs completes the QRA procedure. Therefore, the subject area in which the SI itself should be performed is associated to each activated SI. Through a technical inspection, the expert evaluator must identify the areas that should undergo refurbishment SIs, being expressed by a percentage of the overall gross area. Therefore, the QRA procedure discloses a dataset containing the following:

1. Activated SIs embedding the construction work allocation coefficients into categories.

2. Extensions of areas subjected to each SI.

Thus, the technical assessment is based on a comparison using a set of features defining the deviations between the ideal building situation, based on the current regulations and required standards, and the actual situation. Assessment outputs emerge with an appropriate set of SIs for refurbishment. Each activated SI contains two attributes:

1. Intensity: a percentage that estimates the work charge for a complete refurbishment of the building sub-system that is involved in the SI.

2. Extension of the subject areas involved in the SI.

The intensity, multiplied by the extension, defines the entity of an activated SI.

\subsection{Refurbishment Cost Valuation}

The refurbishment cost valuation (Target B) is based on the QRA procedure's output: activation of SIs with specific construction work categories.

The methodology for intervention cost estimation involves the following:

1. Collecting technical and economic data related to previous refurbishment interventions con-ducted on hospital and healthcare facility buildings: Historic Intervention Database Population (HIDP). 
2. Selecting a scheduled Historic Intervention (HI) to identify the interventions that are similar to the subject building: Case Based Reasoning (CBS).

3. Data elaborations establishing the similarity between the interventions conducted on the buildings identified in phase B.2 and the interventions to be carried out on the subject, characterized by the QRA procedure's results.

\subsubsection{HIDP (Subroutine B.1)}

A database on previous health care facility refurbishments, adjustments, and modernizations is needed to perform a CBR analysis. These interventions must be located in the same geographical context as the subject to hypothesize the comparability of average costs: level NUTS2 in the Nomenclature of Territorial Units for Statistics is considered to be a homogeneous context [77]. Three sections represent the scheduling process, concerning the number of historic interventions for relevant data collection (HIDP). The first one (HIDP.1) involves the entire hospital institution and reports general information, such as reference health authorities, provided services, and buildings that are included in the hospital district (Table 13).

Table 13. General information concerning hospital institution (section HIDP.1).

\begin{tabular}{ll}
\hline \multicolumn{1}{c}{ Type of General Information } & \multicolumn{1}{c}{ Examples/Notes } \\
\hline HIDP.1.1. Local Health Department & Corresponding to the health government/authority \\
HIDP.1.2. Health facility & Corresponding to the hospital/health institution's denomination \\
HIDP.1.3. Typology of hospital & Mono-block, Poly-block, Backbone, Pavilions, etc. \\
HIDP.1.4. Main period of construction & The intended period in which the hospital was operationalized \\
HIDP.1.5. Buildings & Offices, Laboratory, Operational Units, etc. \\
HIDP.1.6. Provided services & Administration, Emergency, Hospitalization, Specialization Services, etc. \\
\hline
\end{tabular}

In the second phase (HIDP.2), the collected information is essential for performing the economic valuation, as this process highlights the parameters for comparable intervention selection. HIDP.2 includes the synthesis of previous healthcare facility interventions, in the NUTS2 context, and collects the relevant features of each refurbishment intervention (Table 14). The availability of each intervention's technical and economic information attributes costs to specific construction work categories.

Table 14. Scheduling features concerning each comparable historic intervention (section HIDP.2).

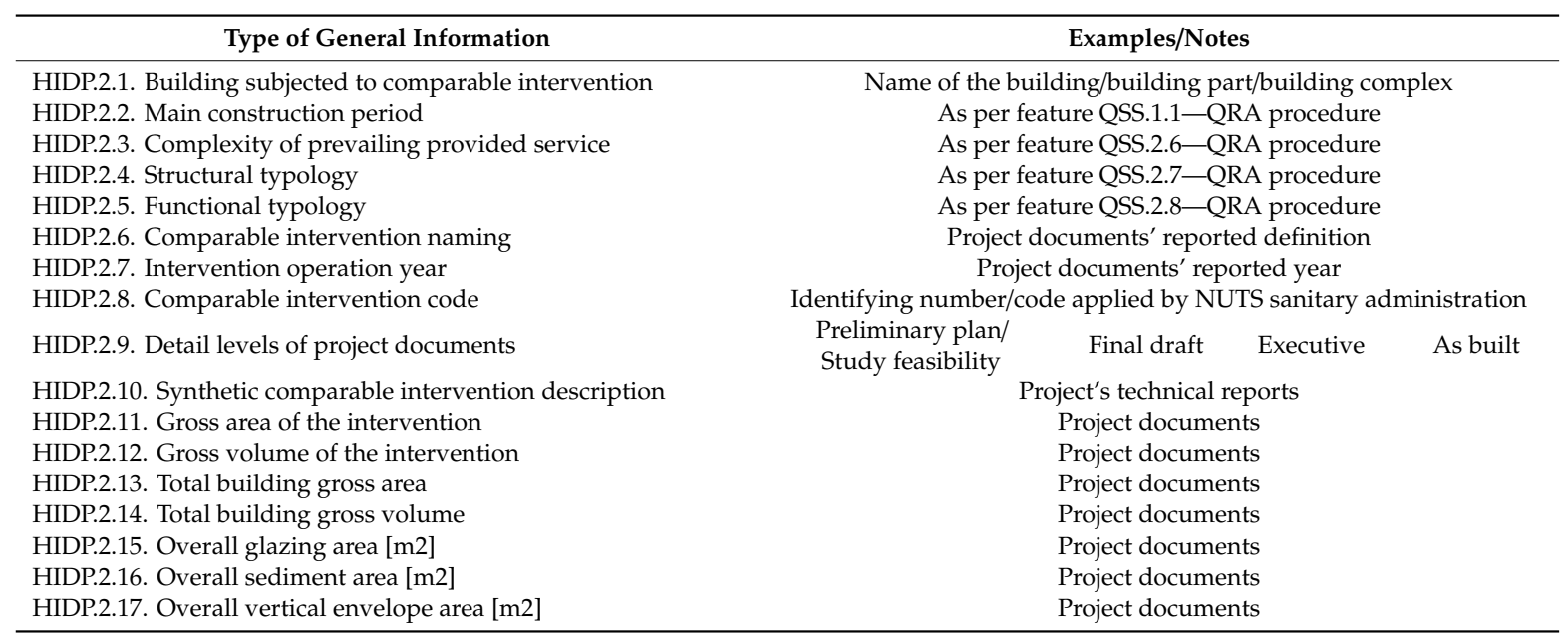

In the third phase (HIDP.3), according to the definitions reported in Tables 6-9, each construction work category is attributed an SI that better complies with the effective actions conducted on the building and the extension of each relevant category, expressed by the percentage of the gross area that is subjected to the intervention. 


\subsubsection{CBS (Subroutine B.2)}

It is possible to perform an Economic Valuation once the HIDP is complete and a suitable intervention is determined (QRA). First, a CBS must be performed. The variables used to identify the subject intervention and the comparable items with known costs aim to describe all of the factors influencing the intervention cost. The CBS is conducted with reference to the features evaluated in the HIDP, particularly HIDP.2.2, HIDP.2.3, HIDP.2.4, and HIDP.2.5, whose possible values are defined in the QSS (Tables 2 and 3). These features are selected due to their influence on refurbishment costs and their ability to select comparable items with the most similar features as the subject.

The binary asymmetric similarity coefficients that we used to perform the comparison are the Jaccard coefficient [21] and the Sørensen coefficient [22]. We chose asymmetrical coefficients, because, if we chose a set of characteristics not present in the evaluated building as the descriptors, this list would be greater, and the more symmetrical coefficients would tend to be 1 . This would distort the real degree of similarity between the subject and the various comparable items. If the Jaccard coefficient and Sørensen coefficient averages are greater than 0.5 , then the intervention is assumed to be a good comparison, otherwise the following steps would exclude it. The quantity and quality of available data scheduled during HIDP could gradually raise this threshold.

\subsubsection{Final Cost Valuation (Subroutine B.2)}

After the CBS, we performed the Final Cost Valuation (FCV), which involves the valuation of similarities between the interventions selected as comparable items and the subject whose refurbishment intervention must be evaluated. We identified a series of descriptors to define a complex intervention and construction work categories (Table 6) to perform the QRA procedure.

We assumed the following parameters:

$e_{j k} \quad$ is the extension interested in the $j$-th construction work category, expressed as a percentage of the overall $k$-th building comparable item's gross area [\%].

$i_{j k} \quad$ is the intensity of the $j$-th construction work category, expressed as a percentage ratio of the $k$-th building (comparable item) same category's corresponding intensity in the new construction [\%].

$\mathrm{C} u_{j}$ is the average unit market prices for the $\mathrm{j}$-th work category applied to the new construction, expressed in the pertinent unit of measure [€/u.m.] (Table 6).

$E_{j k} \quad$ is the entity of the $j$-th work category referred to the $k$-th comparable [-].

$S_{0 k, l}$ is the $l$-th similarity coefficient between the valuation object 0 (subject) and the $k$-th comparable [-].

$c e_{k}$ is the entitary cost of the $k$-th comparable [€/entity].

$\mathrm{ce}_{l}$ is the entity cost of the subject building's refurbishment intervention calculated by the $l$-th similarity coefficient [€/entity].

$A_{0} \quad$ is the subject building's total area $\left[\mathrm{m}^{2}\right]$.

$I_{0} \quad$ is the total intensity of the refurbishment work for the subject building [\%].

$C$ is the total cost of the subject building's refurbishment [€].

The average unit market costs for new constructions $C u_{j}$ are easily derived from direct and indirect sources, applicable to the NUTS2 reference level. Each descriptor is valued by the entity $E_{j k}$, which is defined as the product between the extension $e_{j k}$ of the $j$-th work category. It refers to the $k$-th comparable and it is expressed in percentage terms-considering the total area of the building as $100 \%$-multiplied with its intensity $i_{j k}[\%]$ to the average unit market prices, referring to new buildings for the specific processing category $\mathrm{Cu}_{\mathrm{j}}[€ /$ u.m.]:

$$
E_{j k}=e_{j k} \cdot i_{j k} \cdot C u_{j}
$$

The intensity $i$ is related to the same $j$-th category in refurbishment interventions as compared to the $j$-th category applied to the new construction. The product among the extension of a work category, its intensity, and its average unit cost for the new construction is called the entity. Thus, each descriptor 
will be valorized by its entity. The entity varies between 0 and 1.6, depending on the specific construction work category, as we assume that the processing of some work categories could be more expensive for refurbishment than new construction. We estimate the similarity of the valuation object (subject) with respect to each comparable item according to the entity of each descriptor (each work category involved in refurbishing the building).

The quantitative similarity coefficients chosen for this step are the Steinhaus, Rudjichka, Kulczynski, Jaccard, Dice, and Cosine coefficients.

For example, with reference to the Steinhaus coefficient, the application becomes the following:

$$
S_{0 k, 1}=\frac{2 \sum_{j=1}^{n} \min \left(E_{j 0}, E_{j k}\right)}{\sum_{j=1}^{n} E_{j 0}+E_{j k}}
$$

Each $l$-th similarity coefficient is used to weigh the costs of the selected comparable item $c e_{l}$ interventions in the following way:

$$
c e_{l}=\frac{\sum_{k=1}^{m} S_{0 k, l} \cdot c e_{k}}{\sum_{k=1}^{m} S_{0 k, l}}
$$

The obtained result is used in order to estimate the total cost for the modernization of the valuated health facility subject by multiplying the total cost calculated by each $l$-th similarity coefficient for the area and the overall intensity of the intervention:

$$
C=\frac{\sum_{l=1}^{p} c e_{l}}{p} \cdot A_{0} \cdot I_{0}[€]
$$

To perform the calculation, we need to evaluate $I_{0}$. This parameter is calculated, as follows:

$$
I_{0}=\frac{\sum_{j=1}^{n}\left(C u_{j} \cdot A_{0, j} \cdot \delta e_{j} \cdot \delta i_{j}\right)}{\sum_{j=1}^{n}\left(C u_{j} \cdot A_{0, j}\right)}
$$

where

$\mathrm{Cu}_{j}$ is the average unit market prices for the $j$-th work category applied to new construction, expressed as the pertinent unit of measure [€/u.m.] (Table 6).

$A_{0, j}$ is the appropriate dimension interested in the $j$-th work category [u.m.] (Table 6).

$\delta e_{j}$ is a coefficient that considers the combination of each work category's extensions, embedded in all activated SIs [-] (Tables 5 and 7-9).

$\delta i_{j}$ is a coefficient that considers the combination of each work category's intensities, embedded in all activated SIs [-] (Tables 5 and 7-9).

The evaluation of $I_{0}$ is based on the SI and the related construction work categories. Whenever the QRA procedure assesses the activation of more than one $\mathrm{SI}$, it is possible that the same construction work category would activate within two or more SIs. Thus, it is necessary to combine activated Sis in order to avoid the double counting effects on costs related to categories recurring more than twice in the activated SIs.

The coefficient for the extension combination $\delta e_{j}$ is expressed for the $j$-th work category, as follows:

$$
\delta e_{j}=\alpha_{j} \cdot\left(\max _{s}\right)+\left(1-\alpha_{j}\right) \cdot\left(\sum_{s} e_{s}\right)
$$

where $e_{s}$ is the extension of the $s$-th SI [\%], as derived by QRA procedure, and $\alpha_{j}$ considers the overlap of the same $j$-th category in different SIs [-]. 
The $\alpha_{j}$ coefficient is calculated, as follows:

$$
\begin{gathered}
\text { If } \sum_{s} e_{s}<100 \% \alpha_{j}=0.5 \\
\text { If } \sum_{s} e_{s} \geq 100 \% \alpha_{j}=\frac{\arctan \left(\sum_{s} e_{s}\right)^{2}}{\frac{\pi}{2}}
\end{gathered}
$$

The function that gives $\alpha_{\mathrm{j}}$ value has $\sum_{s} e_{S}$ as an independent variable. This function is continuous for $\sum_{s} e_{s}=1$, as shown in Figure 2 .

In this way, if $\sum_{s} e_{s}<1$, then the most probable extension value of the combination of two or more SIs is the average between the maximum extension found among all activated SIs and the simple sum of the extensions. Contrarily, if $\sum_{s} e_{s} \geq 1$, the $\alpha_{j}$ value leans asymptotically to 1 , giving priority to the contribution of the $s$-th SI, in which the $j$-th construction work category has the maximum extension (Figure 2).

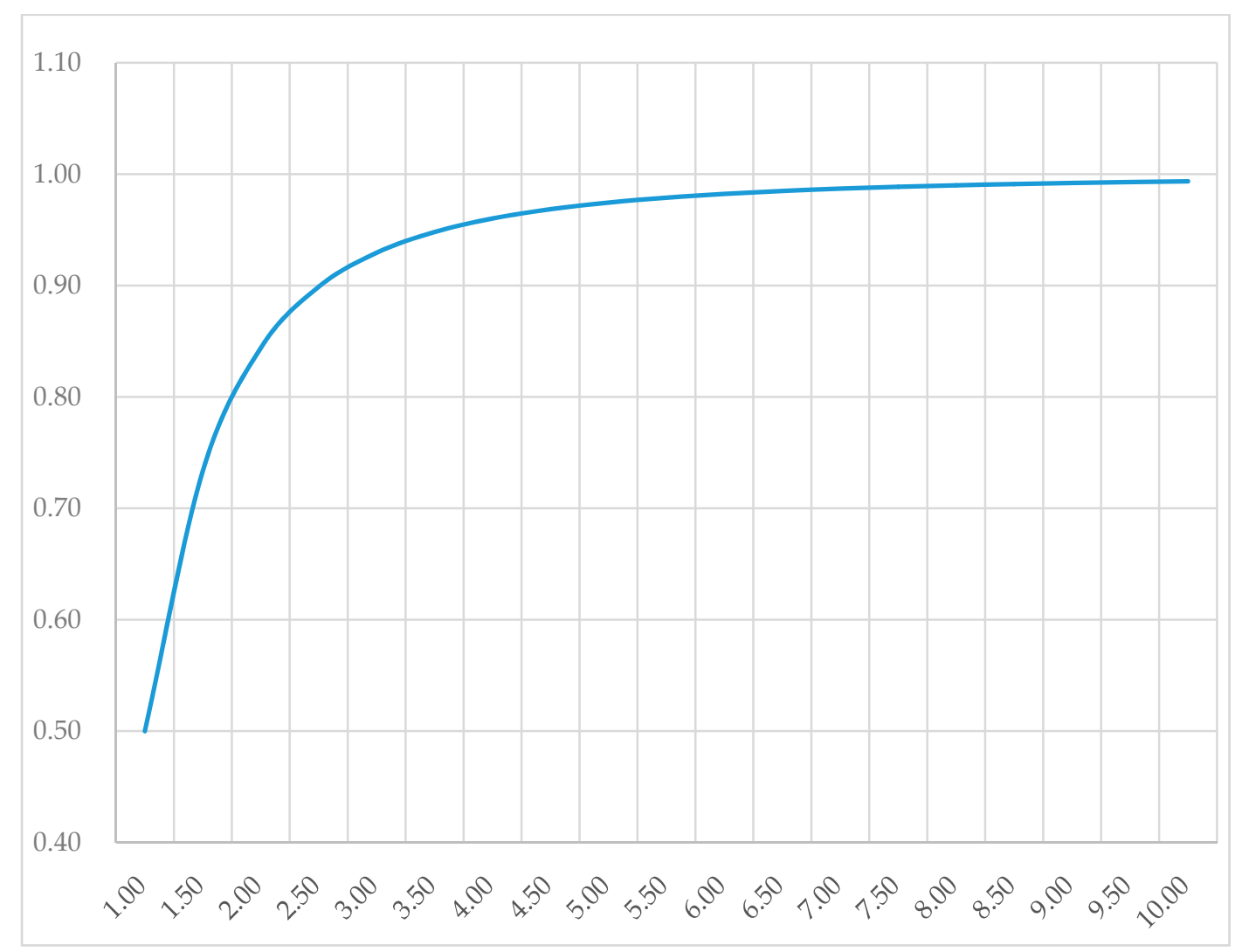

Figure 2. Plotting of $\alpha_{j}$ coefficient values (in ordinates) depending on the $\sum_{s} e_{s}$ value (in abscissae).

The coefficient for the intensities combination $\delta i_{j}$ is expressed, for the $j$-th work category, as

$$
\delta i_{j}=\frac{\sum_{i} e_{s} \cdot i_{j s}}{\sum_{i} e_{s}}
$$

where $e_{s}$ is the extension of the $s$-th SI [\%], as derived by the QRA procedure, and $i_{j s}$ is the intensity of the $j$-th work category in the $s$-th SI [\%].

Thus, for each entity cost derived from the different similarity coefficients, it is possible to obtain a minimum-maximum range value of the total refurbishment cost. 


\section{Case Study}

We applied the proposed model to a case study: pavilion No. 7 'Petracco' of the 'Santa Maria della Misericordia Hospital' in Udine, Friuli-Venezia Giulia region, Italy (Figure 3). This application is a significant test, because pavilion No. 7 has been subjected to a final draft project to bring structural, fire safety, and technical plant adjustments up to regulation, due to the critical issues found in ordinary functionality.

This pavilion was built in 1980, and it consists of four above ground floors and a basement: a typical mono-block type with a load-bearing structure in reinforced concrete. The current use is mainly for patient hospitalization in Neonatal Pathology, Maxillofacial Surgery, and Orthopedics. Tables 15 and 16 reveal the characteristics of the entire hospital and Pavilion No. 7 Petracco.

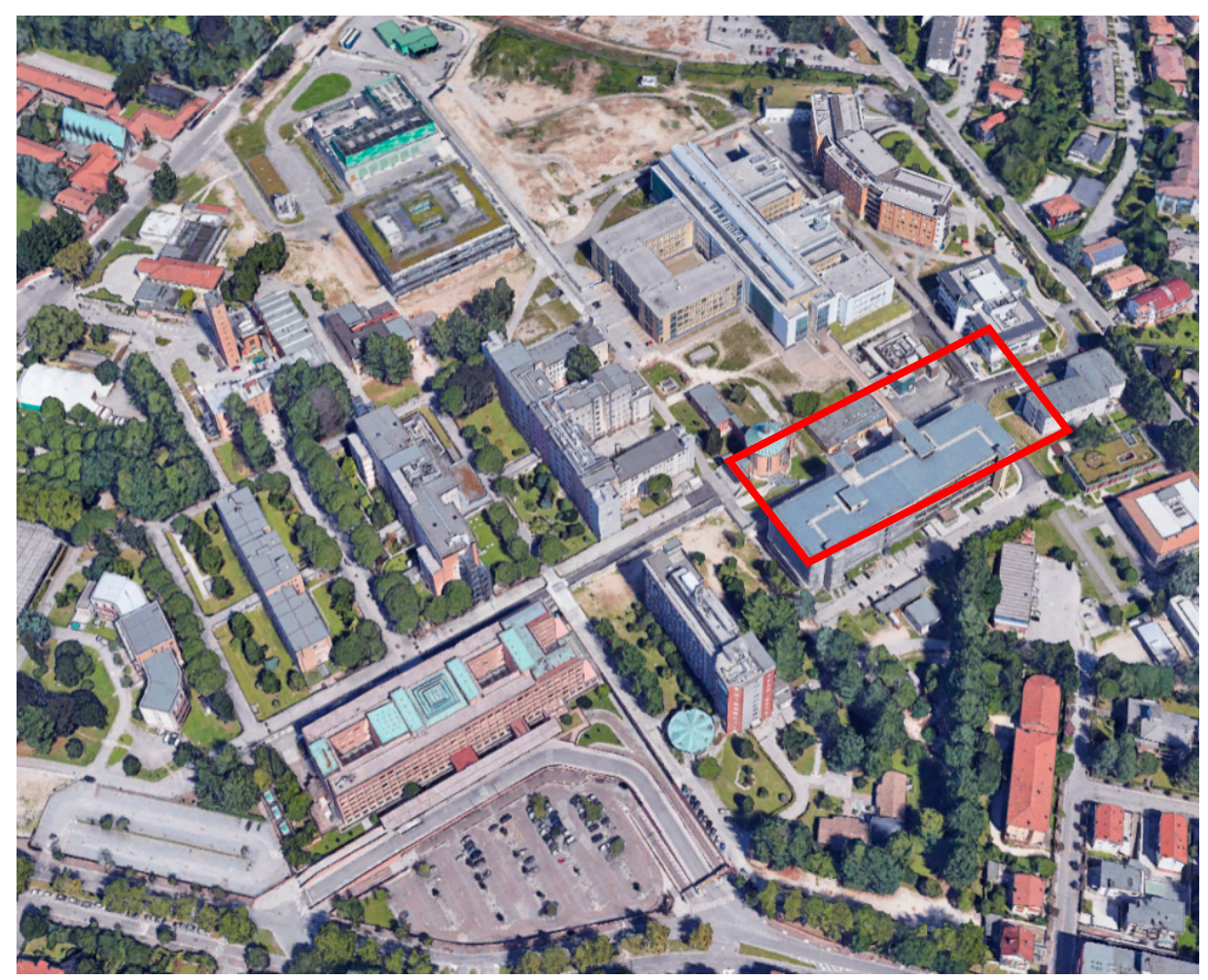

Figure 3. Location of Pavilion No. 7 in the Santa Maria della Misericordia hospital in Udine (satellite view by Google Maps ${ }^{\circledR}$ ).

Table 15. General information concerning Santa Maria della Misericordia hospital in Udine.

\begin{tabular}{ll}
\hline \multicolumn{1}{c}{ Type of General Information } & \multicolumn{1}{c}{ Notes } \\
\hline HIDP.1.1. Local Health Department & ASUIUD-Udine Integrated University Hospital \\
HIDP.1.2. Health facility & Santa Maria della Misericordia Hospital-Udine \\
HIDP.1.3. Typology of hospital & Pavilions \\
HIDP.1.4. Main period of construction & 1924-1938; 1975-1985 \\
HIDP.1.5. Buildings & No. 20 Pavilions \\
& (1) Emergency, (2) Surgical Clinic, (3) Oncology, (4) Internal Medicine, \\
& (5) Anesthesia and Resuscitation, (6) Specialized Medicine, \\
HIDP.1.6. Provided services & (7) Neurological and Neuroscience Clinic, (8) Diagnostic Imaging, \\
& (9) Cardiothoracic Surgery, (10) Transfusion Medicine, (11) Maternal and \\
& Child Department, (12) Laboratory, (13) Administration \\
\hline
\end{tabular}


Table 16. Scheduling subject features concerning each comparable historic intervention.

\begin{tabular}{lc}
\hline \multicolumn{1}{c}{ General Information on Subject Intervention } \\
\hline \multicolumn{1}{c}{ Intervention Features } & Feature Characterization \\
\hline Building subjected to intervention & Pavilion No. 7 'Petracco' \\
Main construction period & $1968-1996$ \\
Complexity of prevailing provided service & Hospitalization \\
Structural typology & Concrete \\
Functional typology & Mono-block \\
Total building gross area $\left[\mathrm{m}^{2}\right]$ & $17,927.03$ \\
Total building gross volume $\left[\mathrm{m}^{3}\right]$ & $90,146.00$ \\
Overall glazing area $\left[\mathrm{m}^{2}\right]$ & 1441.40 \\
Overall sediment area $\left[\mathrm{m}^{2}\right]$ & 3513.00 \\
Overall vertical envelope area $\left[\mathrm{m}^{2}\right]$ & 7939.16 \\
\hline
\end{tabular}

Tables 17-19 report the results of the QSS subroutine applied to Pavilion No. 7, with the valorization of each feature necessary for the SI activation procedure.

Table 17. Results for section QSS.1, concerning Pavilion No. 7.

\begin{tabular}{lcc}
\hline \multicolumn{1}{c}{ QSS-Section QSS.1 } & & \\
\hline \multicolumn{1}{c}{ Features } & Selection & Values \\
\hline QSS.1.1. Construction period & $1968-1996$ & 4 \\
QSS.1.2. Latest intervention for structural adjustments/improvements & $1987-1996$ & 3 \\
QSS.1.3. Latest energy refurbishment of opaque envelope & $1977-1991$ & 2 \\
QSS.1.4. Latest energy refurbishment of transparent envelope & $1977-1991$ & 2 \\
QSS.1.5. Installation period or latest requalification of HVAC systems and plants & $2012-2015$ & 4 \\
QSS.1.6. Installation period or latest requalification of fire-extinguishing and & $2003-2012$ & 3 \\
fire-detection plants & $1971-1990$ & 2 \\
QSS.1.7. Installation period or latest requalification of special and electric plants & $<1997$ & 1 \\
QSS.1.8. Installation period or latest requalification of elevators and lift systems & $2010-2017$ & 3 \\
QSS.1.9. Installation period or latest requalification of medical gas plants & $>1996$ & 3 \\
QSS.1.10. Latest intervention for layout adjustment/architectural barrier removals & $1997-2006$ & 2 \\
QSS.1.11. Latest intervention adjusting the infrastructures for contaminated & & \\
waste disposal and waste storage areas & & \\
\hline
\end{tabular}

Table 18. Results for section QSS.2, concerning Pavilion No. 7.

\begin{tabular}{lcc}
\hline \multicolumn{1}{c}{ QSS-Section QSS.2 } & \\
\hline \multicolumn{1}{c}{ Features } & Selection & Values \\
\hline QSS.2.1. Ground acceleration $\left[\mathrm{m} \mathrm{s}^{-2}\right]$ & $\geq 0.125 \cdot \mathrm{g}$ & 2 \\
QSS.2.2. Heat degree, days [ $\left.{ }^{\circ} \mathrm{C}\right]$ & $2200-2400$ & 2 \\
QSS.2.3. Dry periods, 5-mm-cumulative rainy days per year [mm] & $60-75$ & 2 \\
QSS.2.4. Rainfall intensity [mm] & $55-70$ & 2 \\
QSS.2.5. Activity risk profile & D2 & 5 \\
QSS.2.6. Intended use of the space/area & Hospitalization & 5 \\
QSS 2.7. Structural typology & Concrete & 2 \\
QSS 2.8. Functional typology & Mono-block & 1 \\
\hline
\end{tabular}

Based on the SID and QTE subroutines, Table 20 shows the activated SIs, associated with the relevant extensions and expressed in percentages: the ratio of the overall gross area of Pavilion No. 7 . Thus, all of the activated SIs, characterized by each construction category's intensity and the applied extensions, are available. 
Table 19. Results for section QSS.3, concerning Pavilion No. 7.

\begin{tabular}{lcc}
\hline \multicolumn{1}{c}{ QSS-Section QSS.3 } & & \\
\hline \multicolumn{1}{c}{ Features } & Selection & Values \\
\hline QSS.3.1. Balanced mechanical ventilation & None & 1 \\
QSS.3.2. Renewable energy systems for thermal energy production & None & 1 \\
QSS.3.3. Renewable energy systems for electricity production & None & 1 \\
QSS.3.4. Efficiency of thermal energy production system & $<0.85$ & 1 \\
QSS.3.5. Efficiency of DHW production system & $0.80-0.90$ & 2 \\
QSS.3.6. Dangerous materials in outdoor spaces/areas & Absent & 1 \\
QSS.3.7. Dangerous materials in indoor spaces/areas & Absent & 1 \\
QSS.3.8. Availability of relevant parking areas compared to gross cubic volume $\left[\mathrm{m}^{2} \mathrm{~m}^{-3}\right]$ & $>0.8$ & 4 \\
QSS.3.1. Balanced mechanical ventilation & None & 1 \\
QSS.3.2. Renewable energy systems for thermal energy production & None & 1 \\
QSS.3.3. Renewable energy systems for electricity production & None & 1 \\
\hline
\end{tabular}

Table 20. SIs activated by the QTE subroutine and related extensions.

\begin{tabular}{|c|c|c|c|}
\hline SI Codes & Standard Interventions (SI) & Activation & Extension [\%] \\
\hline SS1 & Global seismic reinforcement & YES & 100 \\
\hline SS2 & Seismic adjustment of HVAC systems and plants & no & - \\
\hline SS3 & Seismic adjustment of sanitary and fire-extinguishing plants & YES & 100 \\
\hline SS4 & Seismic adjustment of medical gas plants & no & - \\
\hline SS5 & Seismic adjustment of special and electric plants & YES & 100 \\
\hline FS1 & New fire compartmentalization/fire protection works & YES & 90 \\
\hline FS2 & Installation of fire-extinguishing hydrant plant/network & YES & 20 \\
\hline FS3 & Installation of fire-extinguishing sprinkler plant/network & YES & 30 \\
\hline FS4 & Sectioning of medical gases network & no & - \\
\hline FS5 & Installation of fire-detection plant & YES & 100 \\
\hline FS6 & Installation of fire escape elevator & YES & 30 \\
\hline LS1 & Improvement of storm water disposal system & no & - \\
\hline LS2 & Flooding protection works & no & - \\
\hline LS3 & Installation of flooding protection pumps & no & - \\
\hline RF1 & Works for architectural barrier removals & no & - \\
\hline RF2 & Functional adjustment of indoor and outdoor spaces & no & - \\
\hline RF3 & Installation/substitution of HVAC systems and plants & no & - \\
\hline RF4 & Modification of HVAC systems and plants & no & - \\
\hline RF5 & $\begin{array}{l}\text { Installation/substitution of sanitary and } \\
\text { fire-extinguishing plants }\end{array}$ & YES & 20 \\
\hline RF6 & Modification of thermal sanitary and fire-extinguishing plants & no & - \\
\hline RF7 & Installation/substitution of medical gas plants & no & - \\
\hline RF8 & Modification of medical gas plants & no & - \\
\hline RF9 & Installation/substitution of special and electric plants & YES & 30 \\
\hline RF10 & Modification of special and electric plants & no & - \\
\hline RF11 & Lift system adjustment & YES & 80 \\
\hline EE1 & Thermal insulation of opaque building envelope & YES & 100 \\
\hline EE2 & Enhancement of transparent building envelope & YES & 100 \\
\hline EE3 & New HVAC systems and plants for higher efficiency & YES & 40 \\
\hline EE4 & New DHW production systems for higher efficiency & no & - \\
\hline EE5 & New power production systems for higher efficiency & no & - \\
\hline ES1 & $\begin{array}{l}\text { Improvement of infrastructures for contaminated } \\
\text { waste disposal }\end{array}$ & no & - \\
\hline ES2 & Improvement of outdoor areas for waste storage & no & - \\
\hline ES3 & Removal of dangerous materials from outdoor spaces & no & - \\
\hline ES4 & Removal of dangerous materials from indoor spaces & no & - \\
\hline ES5 & $\begin{array}{l}\text { Installation of renewable energy system for thermal } \\
\text { energy production }\end{array}$ & no & - \\
\hline ES6 & $\begin{array}{l}\text { Installation of renewable energy system for } \\
\text { electricity production }\end{array}$ & YES & 10 \\
\hline ES7 & Installation of a storage and reuse system for rainwater & no & - \\
\hline FA1 & New parking areas & no & \\
\hline
\end{tabular}


Table 21 reports salient information for CBS procedure according to relevant data collected in HIDP, while Table 22 reports the average score that was obtained in the application of qualitative similarity coefficients to individuate similar interventions. The similarity coefficients' application excludes comparable items No. $4,11,12$, and 15 , as all the qualitative coefficients had a value below 0.5. Moreover, the Dice coefficient had values below 0.5 for all intervention comparable items.

Table 23 reveals entitary results according to Equation (19).

Finally, the cost of refurbishing the assessed building is calculated using an entitary cost (Equation (20)) through the non-zero value averages that were provided by the similarity coefficients.

$$
C=\frac{\sum_{l=1}^{p} c e_{l}}{p} \cdot A_{0} \cdot I_{0}=848.58 \cdot 17,927.04 \cdot 51 \%=7,728,222.31 €
$$

Table 21. Application of Case Based Reasoning (CBS) procedure to the case study: features optioneering.

\begin{tabular}{|c|c|c|c|c|c|}
\hline \multicolumn{2}{|c|}{ Features } & $\begin{array}{c}\text { Construction Period } \\
\text { (HIDP.2.2) }\end{array}$ & $\begin{array}{l}\text { Service Complexity } \\
\text { (HIDP.2.3) }\end{array}$ & $\begin{array}{c}\text { Structural Typology } \\
\text { (HIDP.2.4) }\end{array}$ & $\begin{array}{c}\text { Functional Typology } \\
\text { (HIDP.2.5) }\end{array}$ \\
\hline \multicolumn{2}{|c|}{ Subject } & 1968-1996 & $\begin{array}{l}\text { First Aid/Operating } \\
\text { Rooms/Intensive Care }\end{array}$ & Reinforced concrete & Mono-block \\
\hline \multirow{21}{*}{ 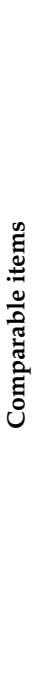 } & 1 & 1968-1996 & Hospitalization & Reinforced concrete & Tower \\
\hline & 2 & 1968-1996 & Hospitalization & Reinforced concrete & Tower \\
\hline & 3 & 1968-1996 & Hospitalization & Reinforced concrete & Tower \\
\hline & 4 & 1900-1938 & Hospitalization & Masonry & Mono-block \\
\hline & 5 & 1939-1967 & Ambulatory clinic & Reinforced concrete & Mono-block \\
\hline & 6 & 1939-1967 & Ambulatory clinic & Reinforced concrete & Mono-block \\
\hline & 7 & 1939-1967 & Laboratory & Reinforced concrete & Mono-block \\
\hline & 8 & 1968-1996 & $\begin{array}{l}\text { First Aid/Operating } \\
\text { rooms/Intensive care }\end{array}$ & Reinforced concrete & Slab \\
\hline & 9 & 1968-1996 & Laboratory & Reinforced concrete & Mono-block \\
\hline & 10 & 1939-1967 & Laboratory & Reinforced concrete & Mono-block \\
\hline & 11 & $<1900$ & Ambulatory clinic & Masonry & Court \\
\hline & 12 & $<1900$ & Ambulatory clinic & Masonry & Court \\
\hline & 13 & 1968-1996 & Ambulatory clinic & Reinforced concrete & Mono-block \\
\hline & 14 & 1968-1996 & Laboratory & Reinforced concrete & Mono-block \\
\hline & 15 & $<1900$ & Ambulatory clinic & Masonry & Court \\
\hline & 16 & 1968-1996 & Hospitalization & Reinforced concrete & Mono-block \\
\hline & 17 & 1968-1996 & Hospitalization & Reinforced concrete & Mono-block \\
\hline & 18 & 1968-1996 & Laboratory & Reinforced concrete & Backbone \\
\hline & 19 & 1968-1996 & Hospitalization & Reinforced concrete & Mono-block \\
\hline & 20 & 1968-1996 & Ambulatory clinic & Steel & Slab \\
\hline & 21 & 1968-1996 & Hospitalization & Reinforced concrete & Tower \\
\hline
\end{tabular}

Table 22. Application of CBS procedure to the case study: individuation of similar interventions.

\begin{tabular}{|c|c|c|c|c|}
\hline \multicolumn{2}{|c|}{ Coefficients } & Jaccard & Sørensen & Average \\
\hline \multicolumn{2}{|r|}{ Subject } & $(1.00)$ & $(1.00)$ & $(1.00)$ \\
\hline \multirow{21}{*}{ 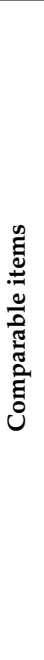 } & 1 & 0.50 & 0.67 & 0.58 \\
\hline & 2 & 0.50 & 0.67 & 0.58 \\
\hline & 3 & 0.50 & 0.67 & 0.58 \\
\hline & 4 & 0.25 & 0.40 & 0.33 \\
\hline & 5 & 0.50 & 0.67 & 0.58 \\
\hline & 6 & 0.50 & 0.67 & 0.58 \\
\hline & 7 & 0.50 & 0.67 & 0.58 \\
\hline & 8 & 0.75 & 0.86 & 0.80 \\
\hline & 9 & 0.75 & 0.86 & 0.80 \\
\hline & 10 & 0.50 & 0.67 & 0.58 \\
\hline & 11 & - & - & - \\
\hline & 12 & - & - & - \\
\hline & 13 & 0.75 & 0.86 & 0.80 \\
\hline & 14 & 0.75 & 0.86 & 0.80 \\
\hline & 15 & - & - & - \\
\hline & 16 & 0.75 & 0.86 & 0.80 \\
\hline & 17 & 0.75 & 0.86 & 0.80 \\
\hline & 18 & 0.50 & 0.67 & 0.58 \\
\hline & 19 & 0.75 & 0.86 & 0.80 \\
\hline & 20 & 0.25 & 0.40 & 0.33 \\
\hline & 21 & 0.50 & 0.67 & 0.58 \\
\hline
\end{tabular}


Table 23. Calculated similarity coefficients and related estimated entitary costs.

\begin{tabular}{|c|c|c|c|c|c|c|c|}
\hline \multicolumn{8}{|c|}{ Similarity Coefficients } \\
\hline & & Steinhaus & Rudjichka & Kulczynski & Jaccard & Cosine & Dice \\
\hline \multirow{16}{*}{ 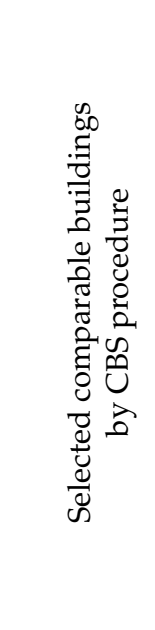 } & $0-1$ & - & - & - & - & 0.54 & - \\
\hline & $0-2$ & - & - & - & - & 0.54 & - \\
\hline & $0-3$ & - & - & - & - & 0.54 & - \\
\hline & $0-5$ & - & - & - & - & 0.72 & - \\
\hline & $0-6$ & 0.55 & - & 0.55 & 0.55 & 0.79 & - \\
\hline & $0-7$ & - & - & - & - & 0.52 & - \\
\hline & $0-8$ & - & - & - & - & 0.73 & - \\
\hline & $0-9$ & 0.53 & - & 0.53 & - & 0.79 & - \\
\hline & $0-10$ & - & - & - & - & 0.73 & - \\
\hline & $0-13$ & - & - & - & 0.54 & 0.74 & - \\
\hline & $0-14$ & - & - & - & - & 0.52 & - \\
\hline & $0-16$ & 0.56 & - & 0.68 & - & 0.58 & - \\
\hline & $0-17$ & - & - & - & - & 0.56 & - \\
\hline & $0-18$ & - & - & - & - & 0.5 & - \\
\hline & 0-19 & - & - & - & - & 0.5 & - \\
\hline & $0-21$ & 0.68 & 0.51 & 0.7 & 0.65 & 0.88 & - \\
\hline \multicolumn{2}{|c|}{$\begin{array}{c}\text { Estimated entitary cost } \\
{[€ / \text { entity] }}\end{array}$} & 836.36 & 1219.89 & 824.60 & 807.64 & 554.40 & - \\
\hline \multicolumn{2}{|c|}{$\begin{array}{c}\text { Average entitary cost } \\
{[€ / \text { entity] }}\end{array}$} & \multicolumn{6}{|c|}{848.58} \\
\hline
\end{tabular}

\section{Concluding Remarks}

The proposed model shows the most probable cost of modernizing buildings. Furthermore, the reliability of the model's result is strongly dependent on the number and quality of available intervention data. The greater the number of codified interventions, the greater the possibility of identifying similar/comparable interventions. It is also possible to subsequently raise the similarity coefficient's threshold for the first and second phase selections. Generally, one must calibrate the threshold for both steps to have a sufficiently broad selection of similar comparable items.

The model's validation is also important. Not much literature and price list data are available, as this study represents a cost valuation for modernizing special purpose facilities. Thus, we tested the model on a case for which refurbishment cost data is available. Starting from Jevons' principle of indifference [6], we investigated the possibility of estimating modernizing costs for health facilities while using similarity coefficients that can weigh intervention costs, as comparable items based on their affinity with the examined building. We assessed coefficient use and their similarities in estimation, but their use is rather limited in this sense.

Overall, we presented a model that identifies modernization needs (technical performance evaluation) and estimates the costs. The first phase, using qualitative coefficients, selects the comparable items according to the building's characteristics, while the second estimates the affinity between the compared and subject interventions using quantitative coefficients. The estimated similarity coefficient weighs the relevant intervention's costs.

We believe that the model offers a good degree of reliability and a quick estimation based on a more detailed procedure when compared to simple parametric valuations. This approach can be used when the cost estimate aims to support the planning of a multi-annual investment budget for buildings refurbishment when they show structural, layout, and plants deficits with respect to current regulations. This tool can estimate the most probable cost of modernizing a special use building and partial interventions that do not concern overall modernization (substituting the performance evaluation). It can also help to estimate a financial budget for planning a series of actions and derive parametric costs for standard interventions. 
The greater issue is the difficulties in finding a quick way to validate the results. In this case, the available refurbishment cost data for the examined building permitted the model's validation. However, future studies should develop a validation that can serve for every application to increase the valuation results' reliability Furthermore, it would be useful to test the model and verify its effectiveness on a greater number of buildings in order to use the results of the assessments to improve its applicability, reliability, and effectiveness.

Author Contributions: Conceptualization, P.R., R.B. and C.A.S.; data curation, C.A.S. and R.B.; formal analysis, C.A.S. and R.B.; funding acquisition, P.R.; investigation, R.B. and C.A.S.; methodology, P.R., R.B. and C.A.S.; project administration, P.R.; resources, P.R., R.B. and C.A.S.; software, R.B. and C.A.S.; supervision, P.R.; validation, R.B., C.A.S. and P.R.; visualization, R.B. and C.A.S.; writing-original draft, R.B. and C.A.S.; writing-review and editing, R.B., P.R. and C.A.S. All authors have read and agreed to the published version of the manuscript.

Funding: This work was supported by the Friuli-Venezia Giulia Region, "ASSIST Project 'Modernization of Health Facilities with the support of Technical and Economic Situational Indicators'" [fund number 2CCPU-ASSISTROSATO-19].

Conflicts of Interest: The authors declare no conflict of interest.

\section{References}

1. Morano, P.; Tajani, F.; di Liddo, F.; Anelli, D. A feasibility analysis of the refurbishment investments in the Italian residential market. Sustainability 2020, 12, 2503. [CrossRef]

2. di Ludovico, M.; de Martino, G.; Prota, A.; Manfredi, G. Empirical damage and repair costs for the assessment of seismic loss scenarios. In XVIII ANIDIS Congress Seismic Engineering; Pisa University Press: Pisa, Italy, 2019.

3. Dell'Anna, F.; Vergerio, G.; Corgnati, S.; Mondini, G. A new price list for retrofit intervention evaluation on some archetypical buildings. Valori Valutazioni 2019, 22, 3-17.

4. Manganelli, B.; Mastroberti, M.; Vona, M. Evaluation of benefits for integrated seismic and energy retrofitting for the existing buildings. In New Metropolitan Perspectives ISHT 2018 Smart Innovation System Technologies; Springer: Cham, Switzerland, 2019; pp. 654-662. [CrossRef]

5. Manganelli, B.; Vona, M.; de Paola, P. Evaluating the cost and benefits of earthquake protection of buildings. J. Eur. Real Estate Res. 2018, 11, 263-278. [CrossRef]

6. Jevons, W.S. The Theory of Political Economy, 3rd ed.; Macmillan and Company: New York, NY, USA, 1888.

7. Choi, S.-S.; Cha, S.-H.; Tappert, C.C. A survey of binary similarity and distance measures. J. Syst. Cybern. Inform. 2010, 8, 43-48.

8. Trobisch, P.D.; Bauman, M.; Weise, K.; Stuby, F.; Hak, D.J. Histologic analysis of ruptured quadriceps tendons, Knee Surgery. Sports Traumatol. Arthrosc. 2010, 18, 85-88. [CrossRef] [PubMed]

9. Hubalek, Z. Coefficients of association and similarity, based on binary (presence-absence) data: An evaluation. Biol. Rev. 1982, 57, 669-689. [CrossRef]

10. Michael, E.L. Marine ecology and the coefficient of association: A plea in behalf of quantitative biology. J. Ecol. 1920, 8, 54-59. [CrossRef]

11. Driver, H.E.; Kroeber, A.L. Quantitative Expression of Cultural Relationships; University of California Press: Berkeley, CA, USA, 1932.

12. Sokal, R.R. A statistical method for evaluating systematic relationships. Univ. Kansas Sci. Bull. 1958, 38, 1409-1438.

13. Smith, J.R.; Chang, S.-F. Automated binary texture feature sets for image retrieval. In Proceedings of the 1996 IEEE International Conference Acoustics Speech, Signal Processing Conference Proceedings, Atlanta, GA, USA, 7-10 May 1996; pp. 2239-2242.

14. Hohn, M.E. Binary coefficients: A theoretical and empirical study. J. Int. Assoc. Math. Geol. 1976, 8, 137-150. [CrossRef]

15. Willett, P.; Barnard, J.M.; Downs, G.M. Chemical similarity searching. J. Chem. Inf. Comput. Sci. 1998, 38, 983-996. [CrossRef]

16. Forbes, S.A. On the Local Distribution of Certain Illinois Fishes: An Essay in Statistical Ecology; Illinois State Laboratory of Natural History: Champaign, IL, USA, 1907.

17. Forbes, S.A. Method of determining and measuring the associative relations of species. Science 1925, 61, 518-524. 
18. Scardi, M. Data Analysis Techniques in Ecology. University of Rome, Department of Biology, 2009. Available online: http://www.michele.scardi.name/corsi/metodi.pdf (accessed on 24 July 2020).

19. Rogers, D.J.; Tanimoto, T.T. A computer program for classifying plants. Science 1960, 132, 1115-1118. [CrossRef] [PubMed]

20. Sneath, P.H.A.; Sokal, R.R. Numerical taxonomy. In The Principles and Practice of Numerical Classification; W.H. Freeman and Company: San Francisco, CA, USA, 1973.

21. Jaccard, P. Étude comparative de la distribution florale dans une portion des Alpes et des Jura. Bull. La Société Vaud. Des. Sci. Nat. 1901, 37, 547-579.

22. Sørensen, T.A. A method of establishing groups of equal amplitude in plant sociology based on similarity of species content and its application to analyses of the vegetation on Danish commons. Biol. Skr. Udg. Det K. Dan. Vidensk Selsk. 1948, 5, 1-34.

23. Motyka, J. O Zadaniach i Metodach badań Geobotanicznych/Sur les buts et les Méthodes des Recherches Géobotaniques; Nakładem Uniwersytetu Marii Curie-Skłodowskiej: Lublin, Poland, 1947.

24. Goodall, D.W. Sample Similarity and Species Correlation. In Ordination of Plant Communities; Whittaker, R.H., Ed.; Springer: Dordrecht, The Netherland, 1978; pp. 99-149. [CrossRef]

25. Kulczyński, S. Die Pflanzenassoziationen der Pieninen; Bulletin de l'Académie polonaise des sciences. Série des sciences biologiques; Imprimerie de l’Université: Krakow, Poland, 1928.

26. Tanimoto, T.T. IBM Internal Report 17th November 1957; IBM Company: Armonk, NY, USA, 1957.

27. Dice, L.R. Measures of the amount of ecologic association between species. Ecology 1945, 26, $297-302$. [CrossRef]

28. Isakson, H.R. The nearest neighbors appraisal technique: An alternative to the adjustment grid methods. Real Estate Econ. 1986, 14, 274-286. [CrossRef]

29. Mahalanobis, P.C. On the Generalized Distance in Statistics. Natl. Inst. Sci. India 1936, 2, 49-55.

30. Isakson, H.R. Valuation analysis of commercial real estate using the nearest neighbors appraisal technique. Growth Chang. 1988, 19, 11-24. [CrossRef]

31. Ciuna, M.; de Ruggiero, M.; Manganelli, B.; Salvo, F.; Simonotti, M. Automated Valuation Methods in Atypical Real Estate Markets Using the Mono-parametric Approach. In Computational Science Its Applications_ICCSA 2017; Gervasi, O., Murgante, B., Misra, S., Borruso, G., Torre, C.M., Rocha, A.M.A.C., Taniar, D., Apduhan, B.O., Stankova, E., Cuzzocrea, A., Eds.; Springer: Cham, Switzerland, 2017; pp. 200-209. [CrossRef]

32. De Ruggiero, M.; Salvo, F. Misure di similarità negli adjustment grid methods. Aestimum 2011, 58, 47-58.

33. Simonotti, M.; Salvo, F.; Ciuna, M.; de Ruggiero, M. Measurements of Rationality for a Scientific Approach to the Market-Oriented Methods. J. Real Estate Lit. 2016, 24, 403-427.

34. Tajani, F.; Morano, P.; Salvo, F.; de Ruggiero, M. Property valuation: The market approach optimised by a weighted appraisal model. J. Prop. Investig. Financ. 2019. [CrossRef]

35. Kulczycki, M.; Ligas, M. Qualitative similarity coefficients in real estate market analysis/Jakościowe współczynniki podobieństwa w analizie rynku nieruchomości. Geomat. Environ. Eng. 2014, 8, 33-41. [CrossRef]

36. Zyga, J. Connection between similarity and estimation results of property values obtained by statistical methods. Real Estate Manag. Valuat. 2016, 24, 5-15. [CrossRef]

37. Zyga, J. Data selection as the basis for better value modelling. Real Estate Manag. Valuat. 2019, $27,25-34$. [CrossRef]

38. OCSE/Osservatorio Europeo Delle Politiche e Dei Sistemi Sanitari. Italia: Profilo Della Sanità 2019; OECD Publishing, Paris/European Observatory on Health Systems and Policies: Paris, France, 2019.

39. Rosato, P.; Alberini, A.; Zanatta, V.; Breil, M. Redeveloping derelict and underused historic city areas: Evidence from a survey of real estate developers. J. Environ. Plan. Manag. 2010, 53, 257-281. [CrossRef]

40. Flores-Colen, I.; de Brito, J.; Freitas, V. Discussion of criteria for prioritization of predictive maintenance of building façades: Survey of 30 experts. J. Perform. Constr. Facil. 2010, 24, 337-344. [CrossRef]

41. Ali, A.; Hegazy, T. Multicriteria assessment and prioritization of hospital renewal needs. J. Perform. Constr. Facil. 2014, 28, 528-538. [CrossRef]

42. Lavy, S.; Garcia, J.A.; Scinto, P.; Dixit, M.K. Key performance indicators for facility performance assessment: Simulation of core indicators. Constr. Manag. Econ. 2014, 32, 1183-1204. [CrossRef] 
43. Dejaco, M.C.; Cecconi, F.R.; Maltese, S. Key performance indicators for building condition assessment. J. Build. Eng. 2017, 9, 17-28. [CrossRef]

44. Rechel, B.; Wright, S.; Edwards, N.; Dowdeswell, B.; McKee, M. Investing in Hospitals of the Future; WHO: Copenhagen, Denmark, 2009.

45. Baraldi, G.; Capurso, L.; Costa, G.; Cuccurullo, F.; di Stanislao, F.; Gensini, G.; Guarini, R.; Mangia, R.; Mauri, M.; Montaguti, U.; et al. Principi guida tecnici, organizzativi e gestionali per la realizzazione e gestione di ospedali ad alta tecnologia e assistenza (Technical, organizational and managerial guiding principles for the construction and management of high technology hospitals). Monitor 2003, 6, 1-296.

46. Head of Italian Government. Approval of Instructions for Hospital Construction; D.G.H. 20 July 1939; Head of Italian Government: Rome, Italy, 1939.

47. Ministry of Public Works, Ministry of the Interior. Limits on Building Density, Height, Distance among Buildings and Maximum Ratios between Residential and Productive Settlements and Public Spaces or Spaces Reserved for Collective Activities, Public Greenery or Parking Areas; M.D. 2 April 1968 No. 1444; Ministry of Public Works, Ministry of the Interior: Rome, Italy, 1968.

48. President of Italian Republic. Approval of the Act of Addressing and Coordination to Regions and Autonomous Provinces of Trento and Bolzano, in Relation to Minimum Structural, Technological and Organisational Requirements for the Exercise of Health Activities; D.R.P. 14 January 1997; President of Italian Republic: Rome, Italy, 1997.

49. Ministry of Public Works. Approval of Technical Standards for Construction in Seismic Zones; M.D. 3 March 1975; Ministry of Public Works: Rome, Italy, 1975.

50. Ministry of Public Works. Technical Standards for Seismic Buildings; M.D. 24 January 1986; Ministry of Public Works: Rome, Italy, 1986.

51. Ministry of Public Works. Technical Standards for the Calculation, Execution and Testing of Structures in Normal and Pre-Compressed Concrete and of Metal Structures; M.D. 9 January 1996; Ministry of Public Works: Rome, Italy, 1996.

52. Prime Minister's Office. Early Features of General Criteria for Seismic Classification of the National Territory and Technical Regulations for Construction in Seismic zone; Ordinance 20 March 2003 No. 3274; Prime Minister's Office: Rome, Italy, 2003.

53. Ministry of Infrastructure. Approval of New Technical Standards for Construction; M.D. 14 January 2008; Ministry of Infrastructure: Rome, Italy, 2008.

54. President of Italian Republic. Standards for Reducing Energy Consumption for Thermal Uses in Buildings; D.R.P. 30 April 1976 No. 373; President of Italian Republic: Rome, Italy, 1976.

55. Italian Parliament. Rules for the Implementation of the National Energy Plan on the Rational Use of Energy, Energy Savings and the Development of Renewable Energy Sources; Law 9 January 1991 No. 10; Italian Parliament: Rome, Italy, 1991.

56. Italian Parliament. Implementation of the 2002/91/EC Directive on Energy Performance in Construction; Lgs. D. 19 August 2005 No. 192.; Italian Parliament: Rome, Italy, 2005.

57. Ministry of Economic Development, Ministry of Environment and Land and Sea Protection, Ministry of Infrastructure and Transportation. Application of Energy Performance Calculation Methodologies and Definition of Minimum Building Requirements; M.D. 26 June 2015; Ministry of Economic Development, Ministry of Environment and Land and Sea Protection, Ministry of Infrastructure and Transportation: Rome, Italy, 2015.

58. Italian Parliament. Implementation of the 2009/28/EC Directive on Promoting the Use of Renewable Energy, with a Change and Subsequent Repeal of the 2001/77/EC and 2003/30/CE Directives; Lgs. D. 3 March 2011 No. 28; Italian Parliament: Rome, Italy, 2011.

59. Italian Parliament. Provisional Nihil Obstat to Activities Subject to Fire Safety Control, Changes to Articles 2 and 3 of the Law 4 March 1982 No. 66, and Supplementary Rules for the Order of the Corpo Nazionale dei Vigili del Fuoco; Law 7 December 1984 No. 818; Italian Parliament: Rome, Italy, 1984.

60. Ministry of the Interior. Approval of the Technical Fire Safety Regulation for the Design, Construction and Operation of Public and Private Health Facilities; M.D. 18 September 2002; Ministry of the Interior: Rome, Italy, 2002.

61. Ministry of the Interior. Update of the Technical Fire Safety Regulation Attached to the Decree of the Minister of the Interior 18 September 2002; M.D. 19 March 2015; Ministry of the Interior: Rome, Italy, 2015.

62. Ministry of the Interior. Code of Fire Safety; M.D. 3 August 2015; Ministry of the Interior: Rome, Italy, 2015.

63. Italian Parliament. Provisions for the Production of Materials, Equipment, Machinery, Electrical and Electronic Installations and Systems; Law 1 March 1968 No. 186; Italian Parliament: Rome, Italy, 1968. 
64. Italian Parliament. Regulations for Plants Safety; Law 5 March 1990 No. 46; Italian Parliament: Rome, Italy, 1990.

65. Ministry of Economic Development. Implementing Regulation of Article 11-Quaterdecies, Paragraph 13, Point b), of Law 2 December 2005, No. 248, about Reordering Provisions of Installation of Technical Systems in Buildings; M.D. 22 January 2008 No. 37; Ministry of Economic Development: Rome, Italy, 2008.

66. Ministry of the Interior. Approval of the Technical Regulation about Fire Safety for Lift Compartments Located in Activities Subjected to Fire Control; M.D. 10 September 1996; Ministry of the Interior: Rome, Italy, 2005.

67. President of Italian Republic. Regulation about Modication to D.R.P. 30 November 1999, No. 162, for the Application of 2014/33/UE Directive on Lifting Plants and Related Safety Components and Operation; D.R.P. 10 January 2017 No. 23; President of Italian Republic: Rome, Italy, 2017.

68. Italian Parliament. Implementation of 93/42/CEE Directive about Medical Devices; Lgs. D. 24 February 1997 No. 46; Italian Parliament: Rome, Italy, 1997.

69. Italian Parliament. Implementation of 2007/47/CE Directive Modifying Directives 90/385/CEE to Bring Member States Laws on Active Implantable Medical Devices Closer Together, 93/42/CE on Medical Devices and 98/8/CE on Biocides Market Entry; Lgs. D. 25 January 2010 No. 37; Italian Parliament: Rome, Italy, 2010.

70. President of Italian Republic. Regulation for Implementation of art. 27 Law 30 March 1971 No. 118 in Favour of the Mutilated and Disabled Civilians, in Terms of Architectural Barriers and Public Transport; D.R.P. 27 April 1978 No. 384; President of Italian Republic: Rome, Italy, 1978.

71. President of Italian Republic. Regulation for the Removal of Architectural Barriers in Buildings, Spaces and Public Services; D.R.P. 24 July 1996 No. 503; President of Italian Republic: Rome, Italy, 1996.

72. Italian Parliament. Implementation of 89/618/Euratom, 90/641/Euratom, 96/29/Euratom e 2006/117/Euratom Directives in Ionizing Radiations; Lgs. D. 17 March 1995 No. 230; Italian Parliament: Rome, Italy, 1995.

73. Italian Parliament. Implementation of 91/56/CEE Directive on Waste, 91/698/CEE Directive on Hazardous Waste and 94/62/CE Directive on Packaging and Packaging Waste; Lgs. D. 5 February 1997 No. 22; Italian Parliament: Rome, Italy, 1997.

74. President of Italian Republic. Regulation on Sanitary Waste Management According to art. 24 Law 31 July 2002 No. 179; D.R.P. 15 July 2003 No. 254; President of Italian Republic: Rome, Italy, 2003.

75. Italian Parliament. Environmental Standards; Lgs. D. 3 April 2006 No. 152; Italian Parliament: Rome, Italy, 2006.

76. President of Italian Republic. Regulation for Design, Installation, Operation and Maintenance of the HVAC Systems in Buildings Aiming at the Reduction of Energy Consumption, in the Implementation of art. 4, Paragraph 4, of Law 9 January 1991 No. 10; D.R.P. 26 August 1993 No. 412; President of Italian Republic: Rome, Italy, 1993.

77. European Commission, Commission Regulation (EC). No. 105/2007 of 1 February 2007 Amending the Annexes to Regulation (EC) No 1059/2003 of the European Parliament and of the Council on the Establishment of a Common Classification of Territorial Units for Statistics (NUTS), Official Journal of the European Union; European Union: Bruxelles, Belgique, 2007.

(C) 2020 by the authors. Licensee MDPI, Basel, Switzerland. This article is an open access article distributed under the terms and conditions of the Creative Commons Attribution (CC BY) license (http://creativecommons.org/licenses/by/4.0/). 